\title{
On the origin of the notching effect during etching in uniform high density plasmas
}

\author{
Gyeong S. Hwang and Konstantinos P. Giapis ${ }^{\text {a) }}$ \\ Division of Chemistry and Chemical Engineering, California Institute of Technology, Pasadena, \\ California 91125
}

(Received 14 June 1996; accepted 15 November 1996)

\begin{abstract}
We present a two-dimensional Monte Carlo simulation of profile evolution during the overetching step of polysilicon-on-insulator structures, which considers explicitly (a) electric field effects during the charging transient, (b) etching reactions of energetic ions impinging on the poly-Si, and (c) forward inelastic scattering effects. Realistic energy and angular distributions for ions and electrons are used in trajectory calculations through local electric fields near and in the microstructure. Transient charging of exposed insulator surfaces is found to profoundly affect local sidewall etching (notching). Ion scattering contributions are small but important in matching experimental notch profiles. The model is validated by capturing quantitatively the notch characteristics and also the effects of the line connectivity and open area width on the notch depth, which have been observed experimentally by Nozawa et al. [Jpn. J. Appl. Phys. 34, 2107 (1995)]. Elucidation of the mechanisms responsible for the effect facilitates the prediction of ways to minimize or eliminate notching. (C) 1997 American Vacuum Society. [S0734-211X(97)02501-8]
\end{abstract}

\section{INTRODUCTION}

Topography evolution during etching of semiconductor devices is critically important in the manufacture of large scale integrated circuits. However, the understanding of the origin of profile peculiarities has lagged behind in the race for ultralarge-scale integration (ULSI). While process recipe development for modern etchers has thus far overcome pattern-dependent etching effects, trial-and-error solutions cannot be relied upon to solve new problems that will arise as targeted device dimensions extend below $200 \mathrm{~nm}$ and aspect ratios exceed 10:1. The "notching" effect ${ }^{1,2}$ is a beautiful example of the unexpected obstacles that could slow down the progress towards realizing ULSI unless fundamental understanding of the mechanisms that affect profile evolution is obtained. Interestingly, the notching effect could have been predicted: a case study that serves to exemplify the power and promise of modeling.

The notching phenomenon ${ }^{1-3}$ describes the opening of a long narrow groove (the "notch") in a conductive material at the interface with an underlying insulator. The notch occurs perpendicularly to the normal ion direction and appears during the overetching step in high density plasma tools. Even more peculiar is the location of the notch: it typically forms at the inner sidewall foot of the outermost feature in a grating-type structure neighboring an open area, as depicted schematically in Fig. 1. The extent of notching depends on the width of the open area between grating structures as well as on the duty cycle of the grating and whether the poly-Si lines are electrically connected. It is also affected by the ion energy distribution, electron temperature, and electron density in the high density plasma employed during the overetching step. Finally, a higher poly-Si conductivity results in more severe notching. ${ }^{3}$ Various scenarios for notch-

${ }^{a}$ Electronic mail: giapis@macpost.caltech.edu ing have been proposed in the literature; all are based on the difference between the potential of the poly-Si line, which attracts excess electrons at the side facing the open area, and the potential at the trench bottom $\left(\mathrm{SiO}_{2}\right)$, which charges up positively. One school of thought suggests that this potential difference causes ion migration to the sidewall foot of the outermost poly-Si line, ${ }^{2,3}$ where they cause localized etching. However, evidence for such surface ion migration on adsorbate (e.g., $\mathrm{Cl}$ ) covered $\mathrm{SiO}_{2}$ is hard to find; furthermore, this mechanism fails to explain the wide notches frequently seen experimentally. A more plausible explanation has been proposed, ${ }^{5,6}$ which attributes notching to electric-fieldinduced distortion of ion trajectories. Local electric fields form as a result of microstructure charging brought about by the directionality difference between ions and electrons at the wafer surface, a mechanism first proposed by Arnold and Sawin. ${ }^{4}$ Ootera et al. ${ }^{5}$ considered the energy distribution of electrons arriving at the microstructure but neglected the ion energy distribution and the effect of the oscillating sheath. Furthermore, they also neglected the conductivity of the poly-Si, thereby "overestimating the local electric field and the ion trajectory deflection.' Steep trajectory bending at the sidewall foot was predicted only for the unrealistic case of zero ion-drift velocity. Kinoshita et al. ${ }^{6}$ improved this picture by assuming a linear sheath model that led to a bimodal ion energy distribution, and by allowing charge redistribution to occur so that the sidewalls of the poly-Si feature reach the same potential. However, the charge was equidistributed along the poly-Si sidewall surface (a physically unrealistic distribution in the presence of the strong positive potential at the trench bottom) which led to the inaccurate calculation of charging potentials and local electric fields. Despite the underestimation of potentials, these authors were the first to point out that the potential difference between the trench space and the poly-Si lines is a maximum for the outermost line. The calculated ion flux distribution at the inner side of 


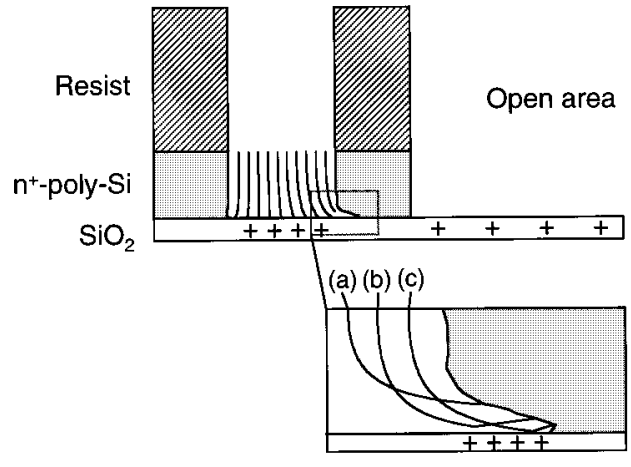

FIG. 1. Schematic depiction of the location of the notch and the mechanisms proposed to contribute to its formation: (a) Ion trajectory bending due to open area charging and direct bombardment of the poly-Si, (b) forward ion deflection due to $\mathrm{SiO}_{2}$ charging under the etched area, and (c) near grazing ion- $\mathrm{SiO}_{2}$ surface collision, followed up by forward scattering and bombardment of the notch apex.

the outermost line was found to peak at the poly-Si/SiO interface; this was suggested to be the reason for notching enhancement at that location. These studies stop short from combining the ion trajectory calculations with a profile evolution simulation to capture the notch formation. Moreover, the calculations pertain to the initial stages of notching and cannot explain why and how the notch can evolve into the narrow wedge, frequently seen in experimental profiles.

We present here a true profile evolution simulation of the notching effect which considers explicitly (a) electric field effects during the charging transient, (b) etching reactions of energetic ions impinging on the poly-Si, and (c) forward scattering effects. Our simulation predicts that notching begins as etching of the poly-Si sidewall foot by energetic ions that get deflected and accelerated towards it. As the notch deepens, more $\mathrm{SiO}_{2}$ surface is exposed and becomes available for forward scattering of ions. In addition, the exposed $\mathrm{SiO}_{2}$ surface charges up causing forward deflection of ions. The contribution of thus scattered or deflected ions becomes increasingly important in defining the notch channel and maintaining the etch rate deep inside the wedge. The proposed mechanisms are schematically depicted in Fig. 1. The significant ion trajectory bending is induced by local electric fields, which form when the microstructure surfaces charge up as a result of the directionality difference between ions and electrons, created by the oscillating electric field in the sheath. The accurate calculation of local electric fields in the microstructure is essential to the modeling of notch profile evolution. The problem is thus divided into three major segments that must be addressed sequentially: (1) A sheath simulation to produce accurate ion and electron energy and angular distribution functions at the microstructure. (2) A charging simulation to determine the steady-state electrostatic force field in and near the microstructure. (3) A notching simulation where ions get deflected and impinge onto poly-Si or $\mathrm{SiO}_{2}$, followed up by reaction (etching) or forward inelastic scattering. These calculations are briefly outlined in the flow chart of Fig. 2 and will be addressed in this article.

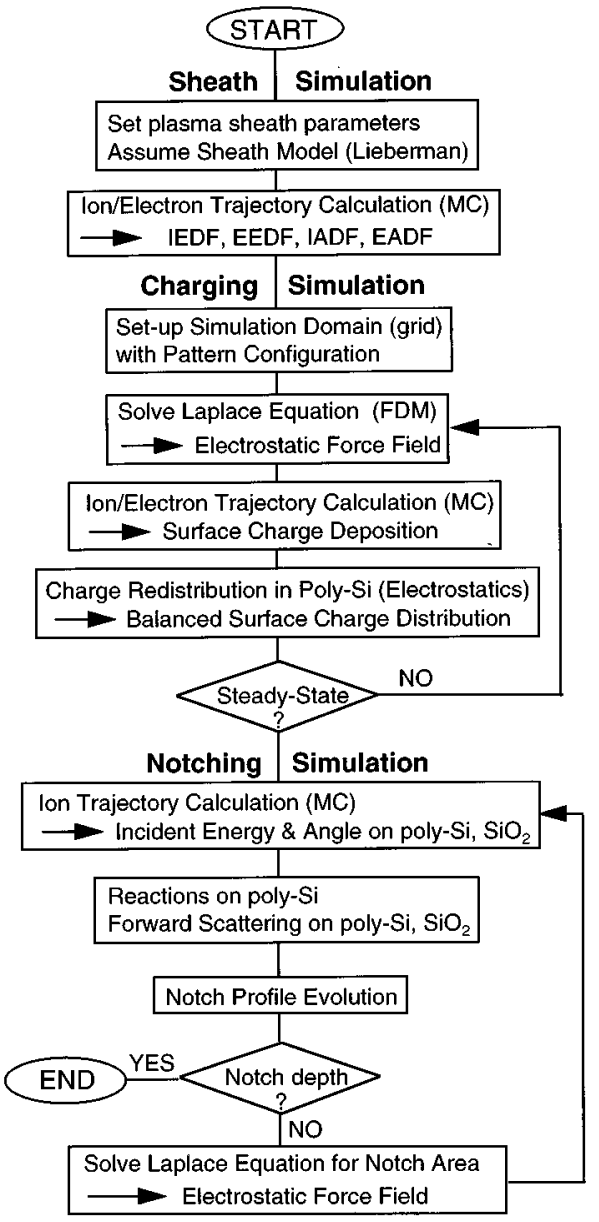

FIG. 2. A schematic flow chart of the simulation steps undertaken in this study.

\section{THE EXPERIMENT OF NOZAWA et al.}

Notching depends on the plasma conditions. In this article, we model the experimental results of Nozawa et al. ${ }^{1}$ They have presented an extensive study of notching in an electron cyclotron resonance (ECR) system using a $\mathrm{Cl}_{2}$ plasma. Their structures consisted of gratings of nominally $0.5 \mu \mathrm{m}$ lines separated by $0.5 \mu \mathrm{m}$ spaces (trenches); the lines were not electrically connected. The gratings were separated by open areas with a width that varied between 0.5 and 10 $\mu \mathrm{m}$. At the onset of overetching, each grating feature consisted of $1 \mu \mathrm{m}$ photoresist onto $0.3 \mu \mathrm{m}$ poly-Si; the features were formed on top of a $0.1 \mu \mathrm{m}$ uniform layer of $\mathrm{SiO}_{2}$ on a $\mathrm{Si}$ substrate. All structures were $200 \%$ overetched at 3.0 mTorr. The mean sheath voltage was estimated to be $37 \mathrm{~V}$, while an rf bias of $60 \mathrm{~V}$ (peak-to-peak) was applied at a rf frequency of $400 \mathrm{kHz}$. In their modeling study of the same problem, Kinoshita et al. ${ }^{6}$ have assumed an electron temperature of $4.0 \mathrm{eV}$ and plasma density of $1 \times 10^{12} \mathrm{~cm}^{-3}$. We will use these reported and estimated values for a direct comparison with the experimental ${ }^{1}$ and modeling results. ${ }^{6}$ 


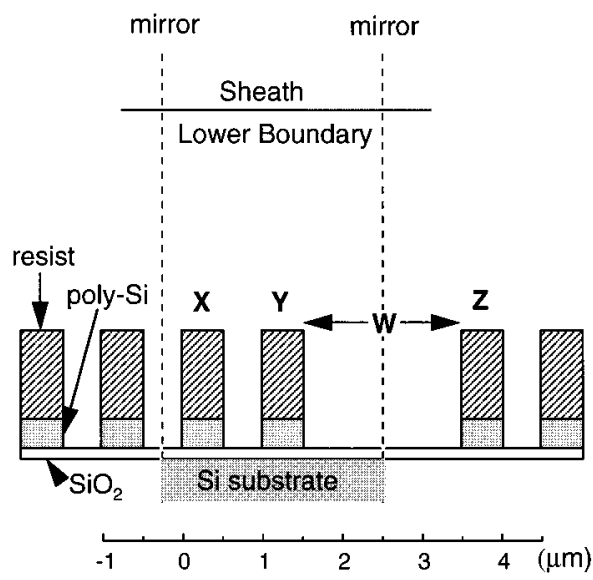

FIG. 3. Schematic depiction of the simulation domain, which is defined by the mirror axes, the sheath lower boundary, and the $\mathrm{SiO}_{2}$ upper surface. Mirror images of the microstructure in the domain are used in calculating the redistribution of charge in the polysilicon lines. The grating lines are 0.5 $\mu \mathrm{m}$ wide, are separated by $0.5 \mu \mathrm{m}$ spaces, and consist of $1 \mu \mathrm{m}$ photoresist onto $0.3 \mu \mathrm{m}$ polysilicon. The width $W$ of the open area is varied in the simulation from 0.5 to $10 \mu \mathrm{m}$. The scale corresponds to the case when $W=2.0 \mu \mathrm{m}$. The sheath lower boundary is $3.7 \mu \mathrm{m}$ above the $\mathrm{SiO}_{2}$ surface.

\section{PLASMA SHEATH AND MICROSTRUCTURE CHARGING SIMULATION}

\section{A. Charging simulation domain}

We begin by defining the two-dimensional (2D) simulation domain as shown in Fig. 3. It occupies a rectangular area, which is $3.7 \mu \mathrm{m}$ in height (sheath lower boundary) and has a variable width between 2 and $12 \mu \mathrm{m}$. The two $0.5 \mu \mathrm{m}$ features $(X$ and $Y)$ in close proximity represent the edge of the grating, while the width of the open area to the next feature $(Z)$ will be varied in order to simulate the effect of larger charge accumulation at the outermost line $(Y)$ of the grating. The notch is expected to appear at the inner side of feature $Y$, just above the poly-Si/SiO electron motion in the simulation domain will be influenced by local electric fields that result from charging of features in the microstructure. Since the charge distribution at the microstructure surfaces uniquely determines the electric field, it is very important to consider the effect of charging potentials outside the simulation domain in determining the charge redistribution in the conductive poly-Si features within the simulation domain. This is accomplished by allowing mirror images of the simulation domain about the left and right boundaries with the same charge distribution.

The simulation domain is discretized by a $2 \mathrm{D}$ grid, which divides the space into a matrix of square cells. The cell dimensions must be small enough to allow for a smooth description of the potential variation in the microstructure. It was found that a resolution of $50 \times 50$ square cells $/ \mu \mathrm{m}^{2}$ was adequate. The grid extends into the dielectric materials and defines a surface layer of cells, where charges will be deposited upon impact of an electron or ion. Thus, the photoresist sidewall surface is divided into 50 cells, while the poly-Si sidewall surface is discretized by 15 cells; each cell has a 20 $\mathrm{nm}$ side along the surface. The mask (photoresist) and the gate oxide $\left(\mathrm{SiO}_{2}\right)$ are both insulators; charge will continue to accumulate in each cell as long as there are particles impinging there. Surface (and bulk) charge mobility is not allowed. On the contrary, the poly-Si has a conductivity high enough to permit charge redistribution or recombination at a timescale shorter than the simulation step. Secondary electron emission is neglected on all surfaces. The $\mathrm{SiO}_{2}$ is assumed to be thick enough to prevent any tunneling current to the substrate. As Kinoshita et al. ${ }^{6}$ have shown, there will be transient charge accumulation at all surfaces until steady state is reached by redirecting ion and electron trajectories. The steady-state potential contour map is essential for ion trajectory calculations during notching and must be determined first.

\section{B. Sheath model}

Ion and electron transport through the sheath must be solved explicitly to determine realistic energy and angularflux distributions of ions and electrons arriving at the microstructure. The energy distributions depend not only on the dc sheath voltage but also on the applied rf bias and rf frequency. The rf electric field in the sheath must be combined with the equation of motion for ions and electrons. Based on a comparison of sheath models, ${ }^{7}$ we have selected the nonlinear model of Lieberman ${ }^{8}$ as the most appropriate for the self-consistent treatment of ion and electron dynamics within the sheath. Using the measured and estimated values for the plasma parameters and bias given in Sec. II, we calculated the average sheath thickness to be $89 \mu \mathrm{m}$. The sheath is assumed to be collisionless in view of the low pressure (3.0 mTorr) used in the ECR plasma. At the aforementioned plasma density, the majority of the ions in the plasma are assumed to be $\mathrm{Cl}^{+}$.

Potentials associated with surface charging are expected to influence the region just above the microstructure. The electric dipole field, formed between the positively charged trench bottom and the negatively charged upper sidewalls, decays very fast with distance. Thus, the electrons and ions crossing the sheath will feel this field only very close to the microstructure. This observation greatly simplifies the simulation because it permits to separately address the problem of ion and electron sheath crossing from the problem of microstructure charging. We will thus solve for the ion and electron energy and angular flux distributions at a plane located a short distance above the microstructure (defined here as the "sheath lower boundary") and then use these distributions to model the surface charging transient. The distance used was $3.7 \mu \mathrm{m}$ above the $\mathrm{SiO}_{2}$ surface; at this height, the surface charging potential decays to zero (vide infra). This distance is indeed very small compared with the calculated sheath thickness of $89 \mu \mathrm{m}$.

The Monte Carlo (MC) simulation of electron and ion transport through the sheath is based on a stochastic generation of electrons and ions at the ion sheath-plasma boundary (sheath edge). Both are treated as particles and their trajec- 
tories are followed by numerically integrating the equations of motion in the 2D plane $(x, y)$ :

$$
M \frac{\partial^{2} y}{\partial t^{2}}=q E_{y}(t), \quad M \frac{\partial^{2} x}{\partial t^{2}}=q E_{x}(t),
$$

where $M$ and $q$ are the mass and the charge of the particle, and $E_{y}(t)$ and $E_{x}(t)$ are the parallel and transverse components of the electric field, defined with respect to the wafer surface normal. While there is no transverse electric field in the sheath, these equations are also used for trajectory tracing near and within the microstructure, where $E_{x}$ is significant. The plasma is assumed to be uniform. The electrons are inertialess and respond to the instantaneous electric field. Electrons enter the sheath upper boundary with an isotropic flux distribution and are assumed to have a Maxwellian velocity distribution with $k_{B} T_{e}=4.0 \mathrm{eV}$, where $k_{B}$ is the Boltzmann constant and $T_{e}$ is the electron temperature. The ion sheathplasma boundary is stationary and ions enter the sheath with initial parallel velocity determined from a Gaussian with a mean equal to the Bohm velocity, $\left(k_{B} T_{e} / M_{i}\right)^{1 / 2}$, and a variance equal to $\left(k_{B} T_{i} / M_{i}\right)^{1 / 2}$, where $M_{i}$ is the ion mass and $T_{i}$ is the ion temperature. Values of $k_{B} T_{i}$ between 0.2 and 0.5 $\mathrm{eV}$ have no observable effect on microstructure charging. In the present simulation, we assume $k_{B} T_{i}=0.5 \mathrm{eV}$; this high value alludes to a collisional broadening of the ion angular distribution as the ions pass through the presheath region and is consistent with recent experimental measurements in similar high density plasmas. ${ }^{9}$ The ions thus enter the sheath with a ratio of directed energy to transverse temperature of 8 .

As a result of the rf oscillation, ions entering the sheath at different phases of the rf cycle will gain different energy as they cross the sheath. At $400 \mathrm{kHz}$, we obtained a bimodal ion energy distribution function (IEDF) for the ions arriving at the sheath lower boundary, shown in Fig. 4(a). The splitting of the ion energy distribution is typical when the sheath thickness is small because ions can cross the sheath in less than one rf cycle. The high energy peak has lower intensity than the low energy peak, as expected from the selfconsistent treatment of the sheath at the low rf bias frequency. ${ }^{7}$ The low energy peak of the IEDF is essential for reaching steady-state charging at the sidewalls. The calculated electron energy distribution function (EEDF) at the sheath lower boundary, shown in Fig. 4(b), is in good agreement with analytic estimates based on the Vlasov equation. ${ }^{5}$ The respective ion angular distribution function (IADF) and electron angular distribution function (EADF) are shown in Figs. 5(a) and 5(b). It is apparent that the IADF is almost unidirectional (the calculated half-width at half-maximum of $4.3^{\circ}$ is consistent with the experimental measurements of Woodworth et al. $)^{9}$ while the EADF is more isotropic at the sheath lower boundary. This directionality difference is critical in facilitating differential charging of the microstructure. ${ }^{4}$ For example, the upper sidewalls of each feature will be preferentially bombarded by electrons, thus developing a negative charging potential. Ions from the "wings" of the IADF are also important because they contribute to the reduction of the negative sidewall potential. However, it must
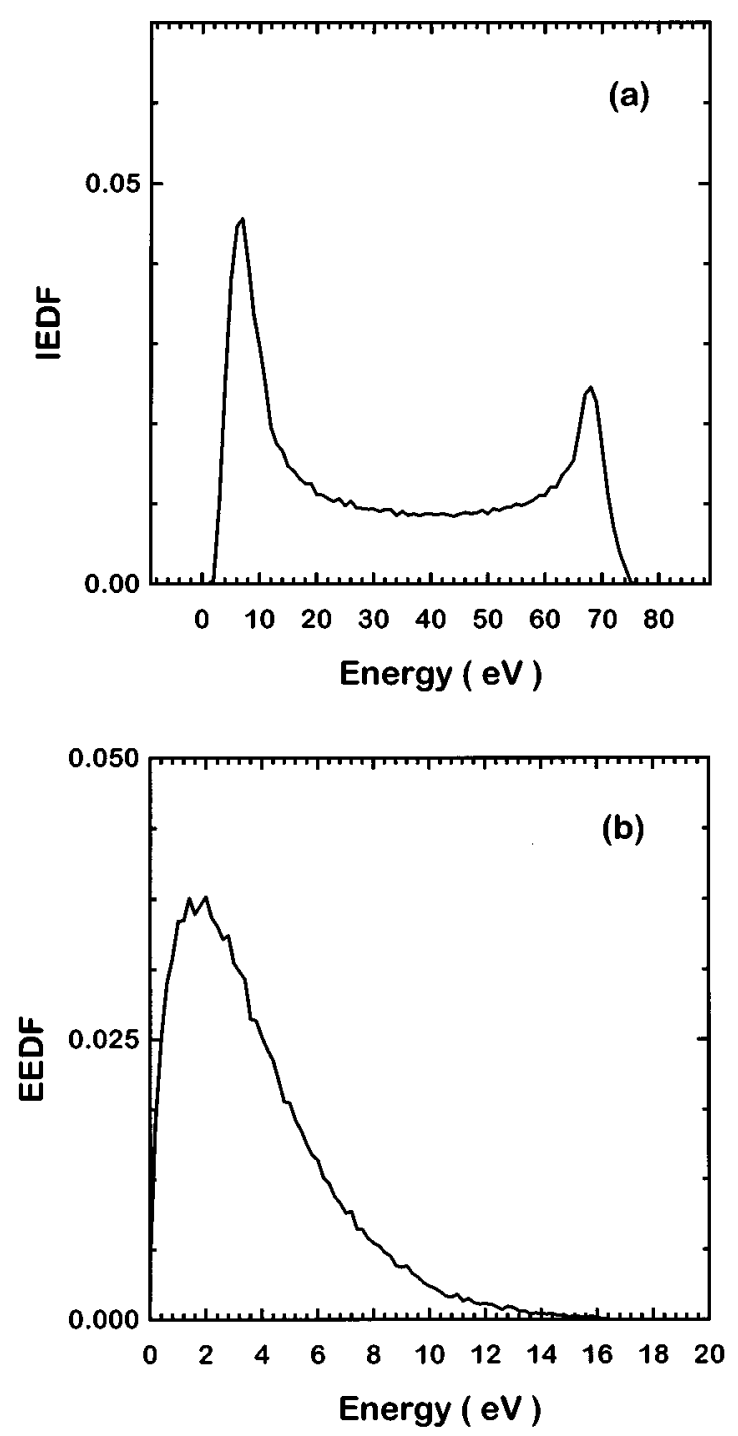

FIG. 4. (a) Ion energy distribution function of $\mathrm{Cl}^{+}$arriving at the sheath lower boundary as predicted by a nonlinear sheath model. (b) The corresponding electron energy distribution function. The plasma parameters were: pressure $3.0 \mathrm{mTorr}$, rf frequency $0.4 \mathrm{MHz}$, rf bias $60 \mathrm{~V}$ (peak-topeak), dc sheath voltage $37 \mathrm{~V}$, electron temperature $4.0 \mathrm{eV}$, and plasma density $1 \times 10^{12} \mathrm{~cm}^{3}$.

be kept in mind that the IADF is energy dependent, that is, the ions that reach the sheath lower boundary at the greatest angles with respect to the etching direction have the least energy.

\section{Charging transient}

Electrons and ions in equal numbers (fluxes) are generated sequentially at the sheath lower boundary having translational energies determined by randomly sampling the corresponding energy distribution functions. Their direction of motion is also determined by random sampling of the corresponding angular distribution functions. Care is taken to account for the energy dependence of the IADF. No etching reactions are considered during the charging transient. Each particle is tracked down through the electrostatic force field 

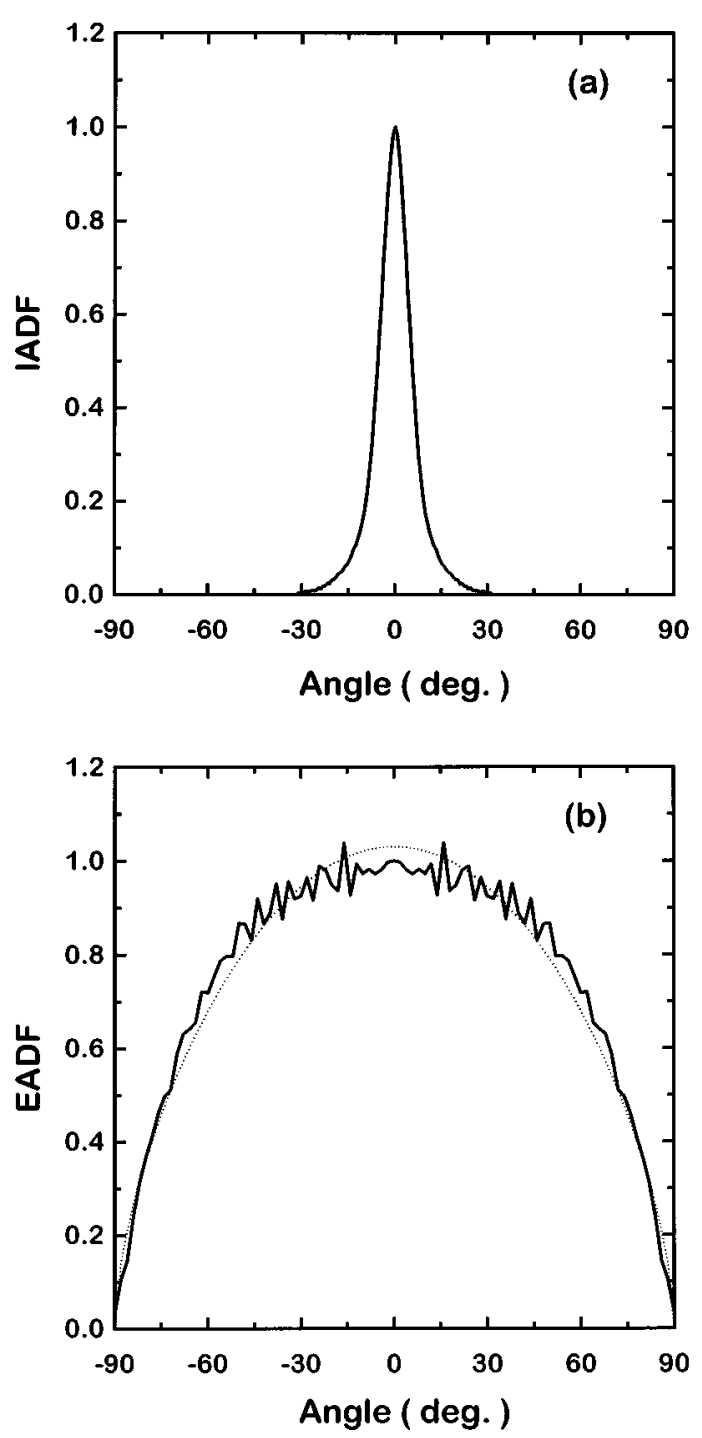

Fig. 5. (a) Ion angular distribution function of $\mathrm{Cl}^{+}$arriving at the sheath lower boundary as predicted by a nonlinear sheath model. (b) The corresponding electron angular distribution function; the dotted line represents a fit by the function $\cos ^{0.6} \theta$. The plasma conditions are given in Fig. 4.

until it impinges onto a surface, where it transfers its charge with $100 \%$ probability. The process by which this charge transfer occurs is not important for the model. The ions probably undergo Auger neutralization before impacting the surface, but the net result is the same as if the ion collided directly with the surface: the surface charge increases by a unit of positive charge. In the initial stages of charging, the electrostatic potentials on the microstructure surfaces are small resulting in weak local electric fields. As more and more particles arrive at different surfaces, charging potentials increase and the electrostatic force field becomes significant enough to alter ion and electron trajectories. For every 50 ions and 50 electrons (the "charging step," corresponding approximately to one-half rf cycle) impacting the microstructure surfaces, the Laplace equation, $\nabla^{2} V=0$, is solved in the entire simulation domain, taking into consideration the modified surface charge distribution along the pattern. The finite differences method (FDM) is employed, with the following boundary conditions: $V=0$ along the sheath lower boundary, $\boldsymbol{\nabla} V=0$ at the left and right boundaries (centerlines); the actual charging potential distributions at the end of each charging step are used for the various microstructure surfaces.

The size of the simulation domain requires a very large number of charging steps before a uniform charge distribution can be obtained. The process is slowed down considerably because a nonuniform charge distribution, typically occurring at the initial stages of charging, results in a "bumpy" electric force field which further affects the distribution of new charges and impedes convergence.

\section{Charge redistribution in the poly-Si}

To obtain the correct surface charging potentials, it was found that the contributions from features in areas neighboring the simulation domain (Fig. 3) are very significant and must be included in the simulation. In particular, charges on the low dielectric constant $(\epsilon=1.6)$ photoresist can influence the charge distribution on the conductive poly-Si even if the photoresist surface is not in direct view of the poly-Si (e.g., top of photoresist, sidewalls above poly-Si, etc.). This requires accounting for electric field effects through dielectric materials. The charges on insulating surfaces are stagnant which simplifies the calculation of their contribution to surface potentials at other surface cells. However, the mobility of charges in the poly-Si line requires a separate treatment of charging at its surfaces. The key condition is that the poly-Si line must be equipotential. Kinoshita et $a l .{ }^{6}$ considered the total net charge in the poly-Si (divided by a constant) as a measure of the surface potential and assumed that the charge is equidistributed along the sidewall surfaces. This assumption is not physical in the presence of strong charging potentials at neighboring insulating surfaces. The surface charge on the poly-Si sidewalls must be unevenly distributed in order to make the surface equipotential. Electron accumulation is enhanced near the poly- $\mathrm{Si} / \mathrm{SiO}_{2}$ interface, a condition that is critically important for the appearance of the notching effect because it affects the potential at the poly-Si and at the trench bottom. The charge redistribution is performed at the end of each charging step in the following manner.

(1) The potential at each surface cell of a poly-Si line is calculated by considering contributions from all charged surface cells, including those at all photoresist and $\mathrm{SiO}_{2}$ surfaces of the simulation domain and its mirror images.

(2) The arithmetic mean of these potentials for both sidewalls of the poly-Si line establishes a deviation from the equipotential for each cell.

(3) An amount of the total surface charge on the poly-Si line proportional to the deviation (normalized by the equipotential) is added to each cell.

(4) Steps (1)-(3) are repeated until all poly-Si surface cells reach the equipotential value.

It can be easily inferred that the poly-Si surface charge distribution peaks near the poly- $\mathrm{Si} / \mathrm{SiO}_{2}$ interface as a result of the large positive potential at the trench bottom. This im- 


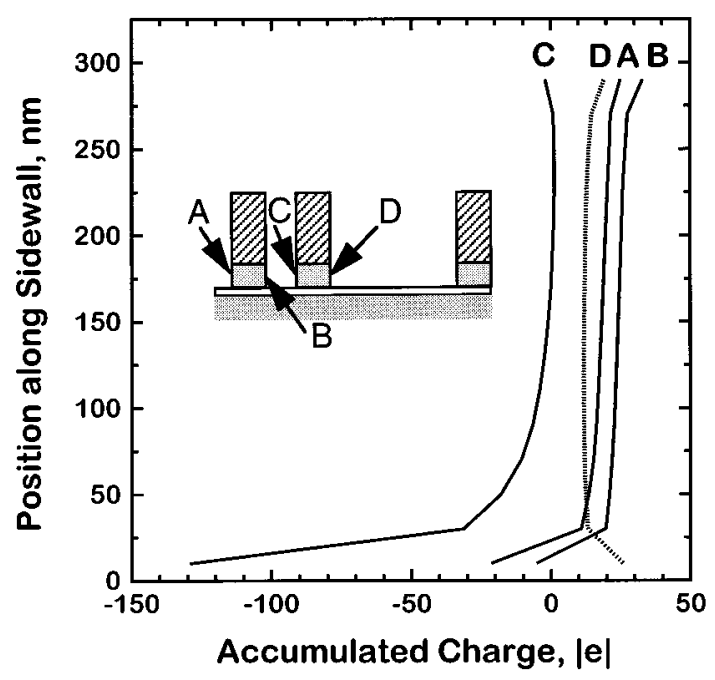

FIG. 6. Distribution of net charge accumulated in each cell ( 1 cell $\equiv 20 \mathrm{~nm}$ ) along the surface of various polysilicon sidewalls (as indicated in the inset) after steady-state charging of the microstructure has been reached. The position of $0 \mu \mathrm{m}$ corresponds to the poly-Si/SiO 2 interface.

portant result is illustrated in Fig. 6 by plotting the net charge accumulated along four poly-Si sidewall surfaces after steady-state charging has been reached in a manner to be described below. Notice the pronounced increase in negative charge in the first cell $(1$ cell $\equiv 20 \mathrm{~nm})$ along the inner poly-Si sidewall of feature $Y$ (curve $C$ ) as compared to the positive charge in the corresponding cell along the outer sidewall (curve $D$ ). The charge redistribution that makes this difference possible is required to make the poly-Si line equipotential. Furthermore, this calculation determines the accurate boundary conditions needed to solve the Laplace equation for the correct 2D potential distribution. At the end, one obtains the modified electrostatic force field at the grid nodes in the space between features and above the microstructure. This field is used to alter particle trajectories as they approach the microstructure during the next charging step.

\section{E. Charging steady state}

The charge redistribution in the poly-Si lines and the solution of the Laplace equation are repeated at the end of each and every charging step until steady state is reached as monitored by surface potential distributions that no longer change. This condition corresponds to equal fluxes of ions and electrons impinging at all points along the microstructure surfaces.

It is instructive to begin the discussion of the charging steady state by monitoring the cumulative electron and ion current density to the combined surfaces of the microstructure in the calculation domain, as well as to the $\mathrm{SiO}_{2}$ surface at the bottom of the last trench before the open area. These current densities are plotted in Fig. 7(a) as a function of the charging step. The ion and electron current densities to all surfaces become equal at a value of 0.965 after about 1500 charging steps (or $750 \mathrm{rf}$ cycles). The currents to the bottom $\mathrm{SiO}_{2}$ surface also balance after the same number of charging
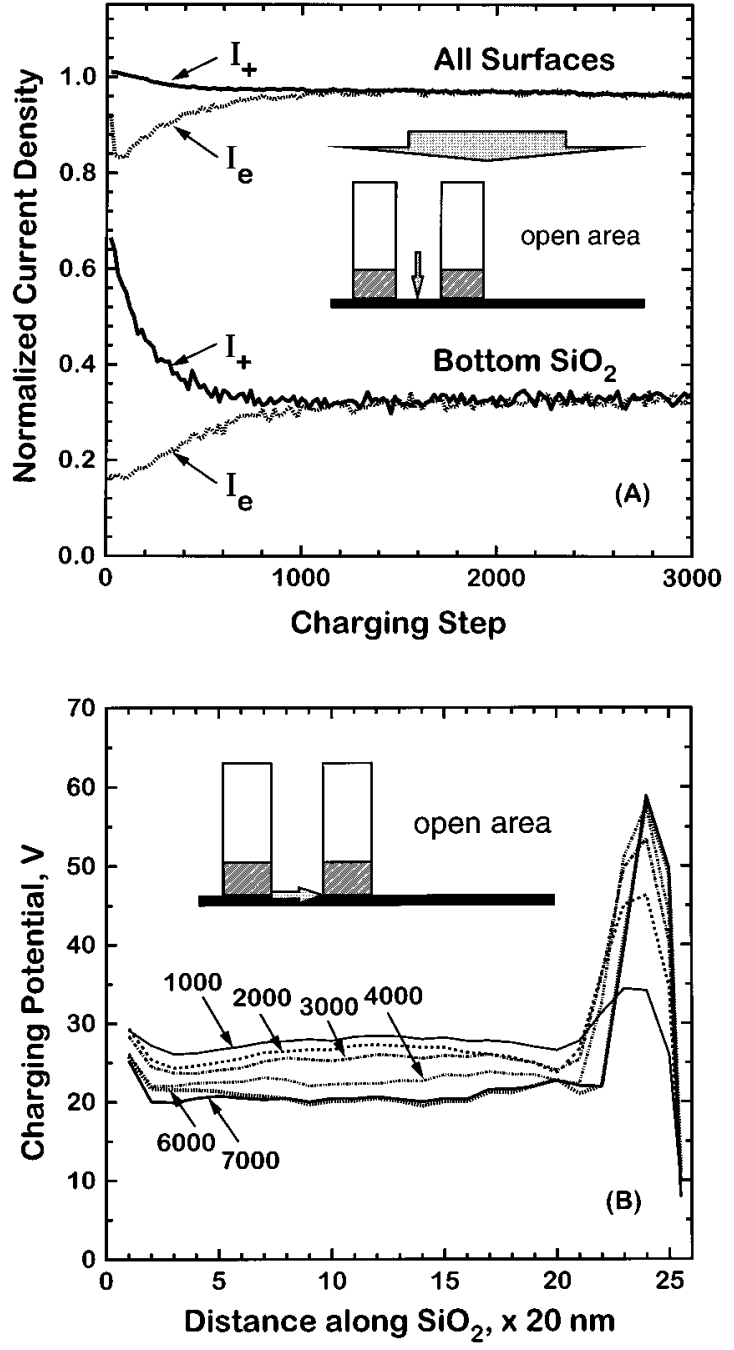

FIG. 7. (a) Normalized ion and electron current densities to all surfaces of the microstructure within the calculation domain and also to the bottom $\mathrm{SiO}_{2}$ surface of the trench nearest to the open area (indicated by the thin arrow), as a function of the charging step. (b) Charging potential distribution along the bottom $\mathrm{SiO}_{2}$ surface of the trench nearest to the open area as a function of the distance along the trench for various counts of charging steps, as indicated. A "charging step" consists of 50 ions plus 50 electrons generated at the lower sheath boundary.

steps. However, equality of the current densities alone does not guarantee that charging has reached steady state. This fact is demonstrated in Fig. 7(b), where the potential distribution along the bottom $\mathrm{SiO}_{2}$ surface of the last trench is plotted at the end of the indicated cumulative numbers of charging steps. The potential distribution becomes progressively very asymmetric, developing a pronounced peak in the vicinity of the inner sidewall foot of the outermost poly-Si line. Clearly, and despite the fact that the ion and electron currents to the bottom $\mathrm{SiO}_{2}$ surface are perfectly balanced [Fig. 7(a)], a dramatic charge redistribution occurs which establishes the electrostatic condition required for ion trajectory bending and acceleration towards the poly-Si sidewall foot. About 7000 charging steps (or 3500 rf cycles) are needed for the potential distribution to reach steady state.

Figure 7 offers insight into how the microstructure 


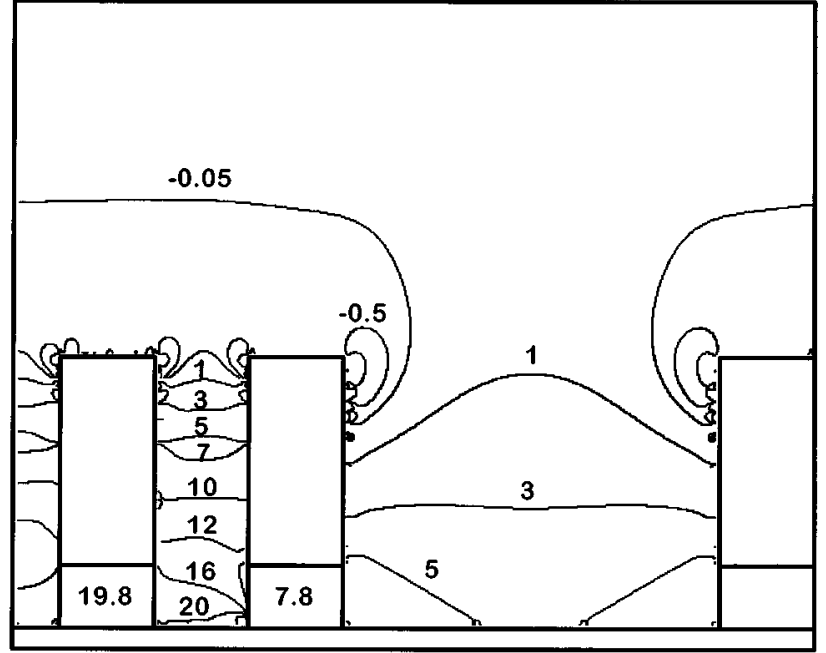

FIG. 8. Potential contour map at steady-state charging. The negative potential above the photoresist decays very fast away from the microstructure due to the electric dipole field formed between the upper photoresist sidewalls and the trench bottom. The curves indicate equipotential lines and the numbers give the values of these potentials in volts.

charges up. At the initial stages of charging and as a result of the directionality difference between electrons and ions, the bottom $\mathrm{SiO}_{2}$ surfaces charge up positively, while the upper sidewalls charge up negatively. As the positive potential at the trench bottom increases, ions with energies at the low end of the IEDF begin to be deflected towards the sidewalls or even out of the trench. The upper sidewall potential becomes less negative allowing more electrons to enter the trench, increasing the electron current to the bottom surface. Electrons that hit the highly conductive poly-Si get redistributed quickly and unevenly to maintain the line at equipotential. The outermost poly-Si line collects more electrons from the open area side which are transferred to the inner side, attracted by the large positive potential at the trench bottom. As the potential difference between the trench bottom and the outermost poly-Si line increases, more ions are steered towards the lower potential, causing a nonuniform potential distribution at the bottom $\mathrm{SiO}_{2}$ surface. The charging steady state corresponds to a dynamic equilibrium where equal positive and negative currents reach all surface segments of the microstructure. Note that, at steady state, about $3.5 \%$ of all ions and electrons that cross the lower sheath boundary never hit the microstructure.

The steady-state charging potential distribution in the microstructure spaces is shown in Fig. 8. This potential contour map is substantially different from the one obtained by Kinoshita et al. ${ }^{6}$ for two reasons: (a) The potential of the poly-Si lines is calculated by allowing electrostatics to determine the charge redistribution in the poly-Si lines; thus, the correct boundary conditions are used in the calculation of the electrostatic force field that affects ion and electron trajectories. (b) We use a nonlinear self-consistent sheath model that results in a quantitatively different IEDF. The asymmetric potential distribution along the $\mathrm{SiO}_{2}$ surface at the bottom of the last trench before the open area [see Fig. 7(b)] is perhaps

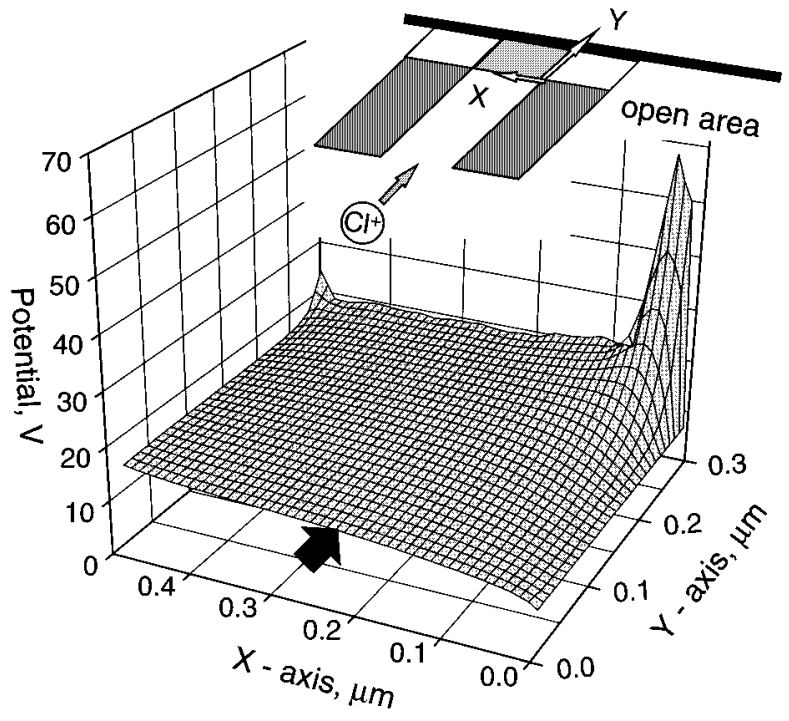

FIG. 9. The 2D potential distribution in the trench area bordered by the poly-Si sidewalls. The inset illustrates the area of interest and defines the origin for the potential surface. The microstructure has been rotated to allow for a more convenient description of ion motion in the trench. The arrows show the direction of ions as they approach the potential surface.

the most dramatic manifestation of the difference between the two studies. Our calculations indicate that the steadystate potential distribution has a pronounced maximum of $58.7 \mathrm{~V}$ very close to the sidewall foot of the outermost poly-Si line (about $40 \mathrm{~nm}$ away); in contrast, Kinoshita et al. show a potential maximum of about $37 \mathrm{~V}$ near the trench centerline [see Fig. 7(a) of Ref. 6]. Given the large potential difference between the two poly-Si lines bordering the trench $(19.8 \mathrm{~V}$ vs $7.8 \mathrm{~V})$, a symmetric potential distribution at the trench bottom is not physical. Electrostatics requires that ions be steered towards the lower equipotential line; the ensuing larger ion flux closer to the outermost poly-Si line leads to a potential maximum significantly removed from the trench centerline.

A clearer picture of why the notch will appear at the foot of the inner sidewall of the outermost poly-Si line emerges from the $2 \mathrm{D}$ potential distribution in the trench space between the poly-Si lines, shown in Fig. 9. An easy way to visualize the influence of this potential surface on ion motion is by analogy to the motion of a marble on an identical topographic terrain within a gravitational field. The peak in the potential distribution thus resembles a sharp "mountain", while the low potential of the poly-Si generates a steep "canyon". A marble (ion) with high initial velocity will reach the end of the terrain (trench bottom). Significantly larger velocities are required to make it uphill. Should an ion approach nearer to the canyon axis $(Y$-axis at $x=0)$ with inadequate velocity to reach the top of the mountain, it will fall downhill into the canyon. The higher uphill it stops in its initial motion, the larger the kinetic energy it will acquire by the time it falls into the canyon (poly-Si sidewall foot). Ions with lower velocity "feel" the slope of the terrain (potential difference between the two poly-Si lines) much earlier and will be diverted towards the front part of the 


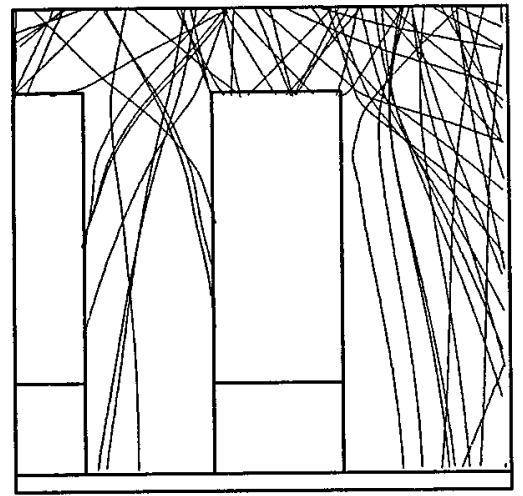

(a)

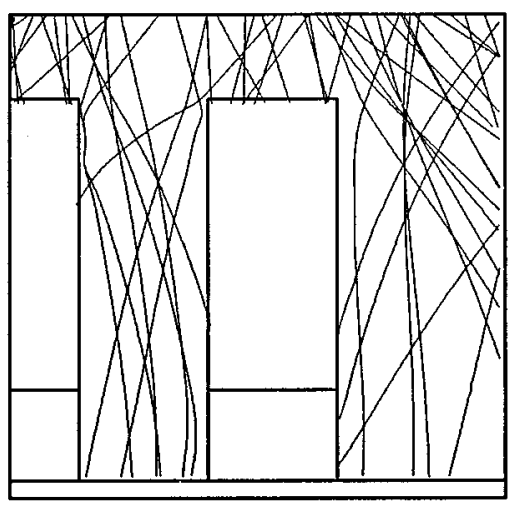

(b)

FIG. 10. Calculated trajectories for (a) $E_{e}=3.0$ and (b) $E_{e}=5.0 \mathrm{eV}$ electrons starting at the sheath lower boundary, after steady-state charging has been reached. Some electrons are deflected back into the plasma. Notice the absence of electron trajectories to the inner sidewall of the outermost poly-Si line. The electron angular distribution has been considered for this calculation.

canyon (upper poly-Si sidewall). Thus, the ions that impinge on the poly-Si sidewall foot will typically have larger energy than ions impinging higher up. Ions that fall on the other side of the "mountain" towards the opposing poly-Si sidewall acquire significantly reduced kinetic energy.

Finally, we briefly discuss what happens at the open area side of the outermost poly-Si line. The poly-Si line acquires a larger potential $(+7.8 \mathrm{~V})$ than the $\mathrm{SiO}_{2}$ surface at the bottom of the open area, an essential condition for electron accumulation at the former. Electrons that approach near the strong repulsive potential of the upper photoresist sidewall get deflected away from the poly-Si sidewall towards the bottom surface. Electrons approaching at oblique angles and at a greater distance from the photoresist stand a better chance of arriving in the vicinity of the poly-Si sidewall, where the higher potential will prevail in attracting them. This establishes a limited window for approaching electrons to reach the poly-Si sidewall. At steady state, the electron current to the outer sidewall is balanced by the ion current to the inner sidewall.

To further validate how steady-state charging affects electron and ion dynamics, we have plotted in Fig. 10 the calculated trajectories for 3.0 and $5.0 \mathrm{eV}$ electrons and in Fig. 11

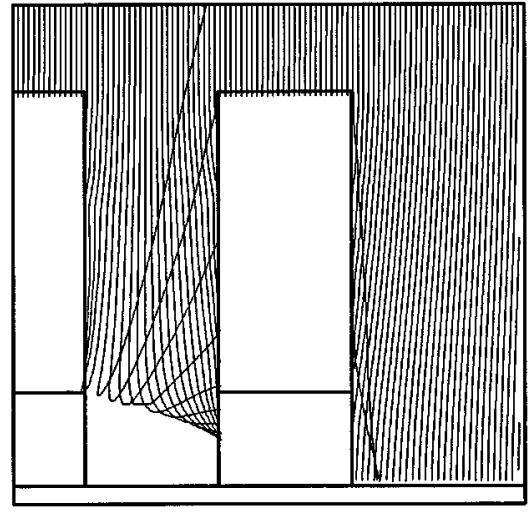

(a)

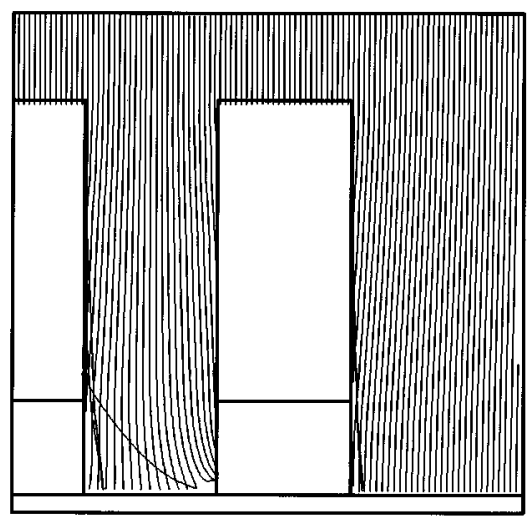

(b)

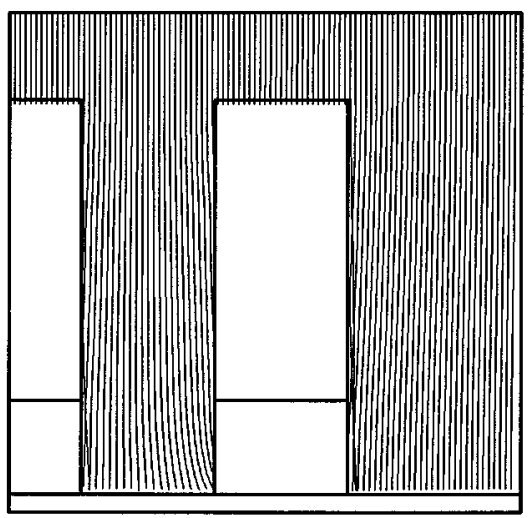

(c)

FIG. 11. Calculated trajectories for ions starting with translational energy of (a) $E_{+}=15$, (b) $E_{+}=30$, and (c) $E_{+}=45 \mathrm{eV}$ at the sheath lower boundary, after steady-state charging of the microstructure has been reached. Notice the deflection of lower energy ions to the sidewalls and out of the trench. Only ions approaching perpendicularly to the wafer are shown.

the calculated trajectories of ions with initial kinetic energies of 15,30 , and $45 \mathrm{eV}$ at the steady-state charging potential. Many low energy $(3.0 \mathrm{eV})$ electrons get deflected at the top of the microstructure; few make it to the bottom of the trenches and more arrive at the bottom of the open area [Fig. 10 (a) ]. On the contrary, more of the higher energy $(5.0 \mathrm{eV})$ electrons make it to the trench bottom and the poly-Si outer sidewall [Fig. 10(b)]. Notice how the electron trajectories get 
deflected from the poly-Si inner sidewall towards the trench bottom. The low energy part of the IEDF is responsible for reaching steady state at the sidewalls. The outermost poly-Si line acquires a larger potential than the neighboring open area (see Fig. 8). Thus, the $15 \mathrm{eV}$ ions get repelled away from the outer sidewall [Fig. 11(a)]. Even higher energy ions are steered away from this surface [Fig. 11(b)]. On the contrary, the larger positive potential at the trench bottom can slow down energetic ions so that they can be deflected and accelerated towards the lower part of the inner poly-Si sidewall [Figs. 11(b) and 11(c)]. The ions that impinge high up along the poly-Si inner surface have reduced translational energy and, thus, will not contribute significantly to etching. Higher energy ions $\left(E_{i} \geqslant 30 \mathrm{eV}\right)$, however, fall down a steeper potential gradient as their trajectories are bent towards the foot of the inner poly-Si sidewall, acquiring a larger transverse energy. There is also some trajectory bending toward the neighboring feature facing the same trench. The key difference between the two sidewalls lies in their charging potential (see Fig. 8). There is a steeper potential gradient of $+50.9 \mathrm{~V}$ over $40 \mathrm{~nm}$ (i.e., $1270 \mathrm{~V} / \mu \mathrm{m}$ ), measured from the potential maximum of $58.7 \mathrm{~V}$ at the trench bottom to the $7.8 \mathrm{~V}$ of the poly-Si line, contrasted with the $+38.9 \mathrm{~V}$ over $460 \mathrm{~nm}$ (i.e., $84.5 \mathrm{~V} / \mu \mathrm{m}$ ) gradient towards the neighboring feature; thus, more energetic ions bombard the former. Significantly, the steeper potential gradient can accelerate ions to transverse energies larger than the threshold for reaction at the poly-Si sidewall (vide infra). Our simulation will reveal that these deflected ions, which impinge directly on the poly-Si sidewall, are responsible only for the initial stages of notch formation.

\section{NOTCH EVOLUTION SIMULATION}

\section{A. Etching reactions and forward inelastic scattering}

When an energetic ion collides with the poly-Si, physical and/or chemical sputtering may take place resulting in materials removal. Collisions with the poly-Si and $\mathrm{SiO}_{2}$ surfaces may also result in inelastic scattering. Reactions and direct inelastic scattering are essential for notching and must be included in the simulation. We employ a simple model of sputtering and scattering, based on previous simulations of profile evolution during neutral beam etching. ${ }^{10}$ When a hyperthermal $\mathrm{Cl}^{+}$impinges on the $\mathrm{SiCl}_{x}$ salvage layer, it will etch with a yield $Y$ that depends on the incident energy $E_{i}$ and angle $\theta_{i}$, as described by the simple formula

$$
\begin{aligned}
& Y=C\left(\sqrt{E_{i}}-\sqrt{E_{\mathrm{th}}}\right) \quad \text { when } \theta_{i}<\hat{\theta}_{\mathrm{cr}}, \\
& Y=C\left(\sqrt{E_{i}}-\sqrt{E_{\mathrm{th}}}\right) \frac{\cos \theta_{i}}{\cos \hat{\theta}_{\mathrm{cr}}} \text { when } \theta_{i} \geqslant \hat{\theta}_{\mathrm{cr}},
\end{aligned}
$$

where $E_{\mathrm{th}}(\mathrm{eV})$ is a translational energy threshold for $\mathrm{Cl}^{+}$ etching of $\mathrm{Si}$ under high $\mathrm{Cl} / \mathrm{Cl}^{+}$flux conditions, $\hat{\theta}_{\text {cr }}$ is a critical angle, and $C$ is a proportionality constant. The square root dependence has been proposed by Steinbrüchel ${ }^{11}$ for physical and ion-enhanced-chemical etch yields at low ion energies. The notching effect is very sensitive to the value of
$E_{\mathrm{th}}$ : a high value generates a miniscule notch or precludes it from occurring and a low value increases the notch shape beyond what is observed experimentally. A value of $E_{\mathrm{th}}=10$ $\mathrm{eV}$ has been recently reported ${ }^{12}$ for the $\mathrm{Cl}^{+}$, etching of $\mathrm{Si}$ under high $\mathrm{Cl} / \mathrm{Cl}^{+}$flux ratio; thus, $E_{\text {th }}$ is not an adjustable parameter in our model. The etch yield is independent of $\theta_{i}$ up to a critical angle of incidence $\hat{\theta}_{\text {cr }}$, typically between $30^{\circ}$ and $60^{\circ}$, beyond which the yield decreases rapidly as forward scattering becomes more important. We hypothesize that such an angle exists for the $\mathrm{Cl}^{+}$etching of $\mathrm{Si}$, based on our results for fluorine scattering at fluorosilyl layers. ${ }^{10}$ However, the notch characteristics were found to be insensitive to the value of $\hat{\theta}_{\text {cr }}$ (between $30^{\circ}$ and $60^{\circ}$ ) as a result of the nature of scattering on the chlorinated poly-Si surface: beyond the initial stages of notching and excluding the apex of the notch, most of the ions impinge at large incident angles, typically $\geqslant 50^{\circ}$, where forward scattering is already significant. For the results presented here, we set $\hat{\theta}_{\mathrm{cr}}=45^{\circ}$. The proportionality constant $C$ affects only the relative rate of notch formation and is not significant for the simulation, as long as it is not excessively large or small. To speed up the calculation, this parameter can be selected so that the most energetic ions of a given IEDF will etch with probability one on normal incidence. For improved importance sampling of the IEDF, all of the results presented here were obtained with $C=0.1$, corresponding to a reaction probability of one for ions with incident energy of $177 \mathrm{eV}$. Negative values of $Y$ signify no reaction. Neither reaction nor sputtering are considered for the photoresist or the $\mathrm{SiO}_{2}$.

Since the poly-Si is heavily $n$-doped $\left(>10^{19} \mathrm{~cm}^{-3}\right)$, there is an appreciable spontaneous etch rate, as high as $5 \mathrm{~nm} /$ min mTorr for etching of poly-Si by $\mathrm{Cl}$ atoms at $0{ }^{\circ} \mathrm{C} .{ }^{13}$ The spontaneous etching will contribute to sidewall etching during both the regular etch and the overetch. Based on the data of Sec. II, we estimated that the sidewall could be etched spontaneously up to $60 \mathrm{~nm}$ by the end of the overetch. Since our simulation does not consider profile evolution during the regular etch, it becomes somewhat arbitrary to include spontaneous etching only during the overetch. We will thus neglect spontaneous etching, expecting a mismatch between the calculated and the experimental profiles. The mismatch should be more pronounced at the upper poly-Si sidewalls (exposed to neutrals for a longer time) and in the notch area, where neutrals get trapped.

Scattering occurs at both the chlorinated poly-Si and the $\mathrm{SiO}_{2}$ surface. Scattering on the photoresist surfaces is not important for notching. Among the different modes of scattering, ${ }^{10}$ only direct inelastic (or single-bounce) scattering is important for notching as a result of the large translational energy of the emerging particles. In spite of the large numbers of ions that undergo multiple-bounce scattering and trapping desorption, these ions do not have large enough energy to induce physical or chemical sputtering in a subsequent collision event and are therefore neglected. Direct inelastic scattering is particularly important at large angles of incidence when the energy transfer to the surface is small. This situation is frequently encountered at the sloping 
poly-Si surface that defines the upper notch boundary, whenever deflected ions impinge directly onto the poly-Si surface. However, only grazing collisions will result in projectiles that subsequently impinge on poly-Si; the vast majority of the directly scattered projectiles will reach the $\mathrm{SiO}_{2}$ surface, where they must scatter again to be able to reach the poly-Si surface. Energy losses in such double bounces reduce the significance of the corresponding scattered projectiles for notching. Thus, direct inelastic scattering on poly-Si $\left(\mathrm{SiCl}_{x}\right.$ layer) need not be considered in a notching simulation.

Direct inelastic scattering on the $\mathrm{SiO}_{2}$ surface can, however, be important as all the ejected projectiles will arrive at the poly-Si surface. Although initially very small, the number of ions that scatter directly on the $\mathrm{SiO}_{2}$ surface increases concomitant with the increase in exposed $\mathrm{SiO}_{2}$ as the notch evolves. Scattering contributes to the notch characteristics and must be included in the simulation. However, it will be shown later that charging of the exposed $\mathrm{SiO}_{2}$ leads to forward deflection of ions without contact with the $\mathrm{SiO}_{2}$, thereby reducing the significance of scattering. In the absence of experimental information on $\mathrm{Cl}^{+}$scattering on a chlorinated $\mathrm{SiO}_{2}$ surface, only specular scattering at incident angles $\theta_{i} \geqslant \theta_{\text {cr }}$ (forward reflection) will be considered in our simulation, with a scattering probability $P_{d}$ given by

$$
P_{d}=\frac{\theta_{i}-\theta_{\mathrm{cr}}}{\pi / 2-\theta_{\mathrm{cr}}}
$$

where $\theta_{\text {cr }}$ is a critical angle, corresponding to the onset of direct inelastic scattering on $\mathrm{Cl}$-covered $\mathrm{SiO}_{2}$, which takes a value between $30^{\circ}$ and $60^{\circ}$. When $\theta_{i} \leqslant \theta_{\text {cr }}$, only multiplebounce scattering and trapping desorption are possible, resulting in scattered projectiles with low translational energy (unimportant for notching). We assume that the charge of the ion is transferred to and remains at the surface upon impact, so that the scattered particle is neutral; thus, its motion is no longer affected by electric fields. We shall also assume that the direct inelastic scattering of $\mathrm{Cl}^{+}$on the $\mathrm{Cl}$-covered $\mathrm{SiO}_{2}$ surface closely follows hard-sphere collision kinematics. Thus, the translational energy $E_{f}$ of the specularly scattered atom will be determined from the equation

$$
\begin{aligned}
\frac{\Delta E}{E_{i}}= & \frac{2 \mu}{(\mu+1)^{2}}\left[1+\cos 2 \theta_{i} \sqrt{1-\mu^{2} \sin ^{2} 2 \theta_{i}}\right. \\
& \left.+\mu \sin ^{2} 2 \theta_{i}\right],
\end{aligned}
$$

where $\Delta E=\left(E_{i}-E_{f}\right)$, and $\mu$ is the ratio of the mass of the gas atom over the effective mass of the surface. Equation (4.3) represents the hard-sphere model prediction ${ }^{14}$ for specular scattering and does not take into consideration surface-atom motion or the gas-surface attractive potential; however, these effects should be negligible for the high incident energies considered here. The notch depth is sensitive to the value of $\mu$, as it will be shown later. For a Cl-covered $\mathrm{SiO}_{2}$ surface, a value of $\mu=1.0$ or slightly lower can be justified.

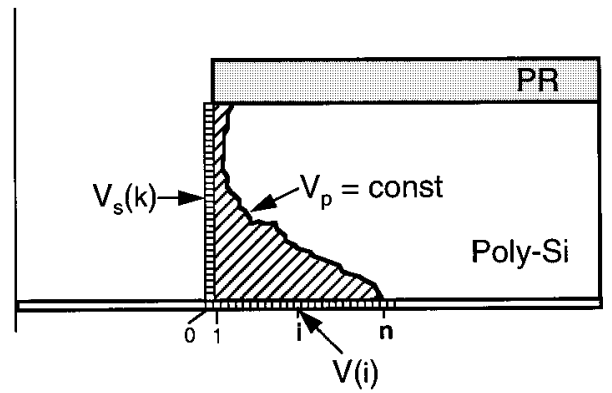

FIG. 12. Definition of the potential distributions in the etched area (crosshatched region) needed for the solution of the Laplace equation to determine the local electric field. The cells depict schematically the discrete nature of the potentials along the bottom surface $[V(i)]$ and along the initial position of the poly-Si sidewall $\left[V_{s}(k)\right]$. The discretization along the moving notch boundary is not shown. The Laplace equation is solved for every new cell position $(i=1, n)$ reached by the notch boundary along the bottom surface as the notch evolves. The photoresist (PR) is not to scale.

\section{B. Forward ion deflection}

As the notch evolves, more $\mathrm{SiO}_{2}$ surface is exposed to ion bombardment resulting in charge accumulation there. The charging alters the local electric field in the etched area (crosshatched region of Fig. 12) and will affect ion motion in the vicinity of the notch. It will be shown later that this local electric field perturbation has a profound effect on the notch shape and depth. However, its inclusion in the simulation poses a formidable challenge, because it requires coupling of the charging and etching calculations. Moreover, charging will not necessarily reach steady state in the vicinity of the notch as the notch boundary evolution is time dependent.

To simplify the calculation, we model this transient charging of the $\mathrm{SiO}_{2}$ surface by assuming that the perturbation is local and does not affect significantly the steady-state electric field in the trench area or the equipotential of the poly-Si line. Thus, the potential boundary conditions at the moving poly-Si boundary $\left(V_{p}=\right.$ const. $)$ and the imaginary line that defines the initial position of the poly-Si sidewall prior to notch formation $\left[V_{s}(k), k=1,60\right]$ can be both obtained from the steady-state charging calculation. The Laplace equation can now be solved for the notch region to determine the modified local electric field, provided that the potential boundary condition at the exposed $\mathrm{SiO}_{2}$ surface is specified. The potential distribution at this surface is dependent on the net charge accumulation. Since the notch is small and shadowed from the plasma, the number of electrons arriving at the exposed $\mathrm{SiO}_{2}$ surface must be small and will be neglected in our calculation. Thus, we shall assume that the potential along the exposed $\mathrm{SiO}_{2}$ surface is proportional to the cumulative ion density there. If $N(i)$ is the total number of ions impinging at cell $i$, where $i=1, n$ and $n$ denotes the cell under the moving poly-Si edge (see Fig. 12), then the boundary condition can be written as

$$
V(i)=V_{p}+\frac{N(i)}{N(1)}\left[V_{0}-V_{p}\right],
$$

where $V(i)$ is the discrete value of the potential at cell $i$, 
$V_{0}=\left[V_{s}(0)+V_{p}\right] / 2$ is the potential at the initial position of the poly-Si sidewall, $V_{p}$ is the potential of the poly-Si, and $V_{s}(0)$ is the potential at the cell just before the poly-Si sidewall. $V_{s}(0)$ and $V_{p}$ are fixed at the final values obtained in the steady-state charging calculation.

As a result of the positive charge accumulation at the the exposed $\mathrm{SiO}_{2}$, lower energy ions approaching its surface get slightly deflected in the forward direction rather than impinge on the $\mathrm{SiO}_{2}$. This effect on one hand reduces the contribution of scattering to notching but on the other hand allows the forward deflected ions to preserve their translational energy by avoiding contact with the surface (an energy loss mechanism); thus, the probability for etching upon subsequent impingement on the poly-Si surface is significantly increased.

\section{Profile evolution methodology}

The etching simulation begins after charging of the microstructure has reached steady state. All results reported in this section are for the electrostatic force field corresponding to the potential contour map of Fig. 8; this field is used to alter the trajectories of ions generated at the sheath lower boundary in identical fashion to the charging simulation. The part of the charging simulation domain that extends from the sheath lower boundary to the photoresist/poly-Si interface is also used for ion trajectory determination, which is performed by integrating Eqs. (3.1). The space below between the poly-Si lines is discretized with a refined 2D grid of square cells, which extends into the poly-Si lines. Smaller cell dimensions are required to delineate the steep profile characteristics; in the limit, each cell can contain a few surface atoms but at a substantial cost to the computational time. We use a resolution of $60 \times 100$ square cells to discretize each poly-Si feature, which corresponds to an area of $25 \mathrm{~nm}^{2} /$ cell. Note that even this denser grid will result in contours that show statistical roughness; however, the profiles can still be captured in reasonable detail. A surface cell is removed when it has been bombarded by a sufficient number of energetic particles (ions and scattered neutrals). For the present calculation, we use 50 reactive collisions to remove each cell; very small numbers (e.g., less than 10) produce rougher surfaces, an artifact of the stochastic nature of the Monte Carlo technique. This procedure corresponds to removing clusters of surface atoms after adequate bombardment, which greatly speeds up the computation.

Ions are generated at the sheath lower boundary with translational energies determined by randomly sampling the calculated IEDF of Fig. 4(a) and angles of approach that are obtained from the corresponding IADF of Fig. 5(a). Ions with initial energy larger than $60 \mathrm{eV}$ do not get bent towards the sidewall; these ions could be neglected to speed up the calculation. When a $\mathrm{Cl}^{+}$impinges at a poly-Si surface, its translational energy and angle of incidence must be known before Eq. (4.1) can be used to determine if reaction will occur. The incident energy is easily obtained from the charging simulation. The incident angle is defined as the angle between the incident trajectory and the normal to the surface at the point of impact. The latter is inferred from the surface slope, which is calculated by a least-squares fitting of the positions of five surface cells about and including the cell where the impact occurs. If reaction does not take place, the $\mathrm{Cl}$ ion will undergo some kind of inelastic scattering. As discussed earlier, these scattered atoms are relatively unimportant for notching since the majority of them impinge subsequently on the $\mathrm{SiO}_{2}$ surface. Thus, all scattered $\mathrm{Cl}$ atoms from the $\mathrm{SiCl}_{x}$ layer could be neglected for the notch evolution.

Forward scattering on the $\mathrm{SiO}_{2}$ surface is accounted for; its influence on the notching effect is relatively small but important. Few energetic ions impinge on the bottom $\mathrm{SiO}_{2}$ surface at large angles of incidence [Figs. 11(b) and 11(c)] during the initial stages of notching. However, the evolution of the notch exposes more $\mathrm{SiO}_{2}$ surface area, thereby increasing inelastic scattering. As a result of the large incident angles involved, such collisions at the $\mathrm{SiO}_{2}$ surface lead to the ejection of $\mathrm{Cl}$ atoms with large translational energies in the forward direction. Scattered $\mathrm{Cl}$ atoms impinge on the evolving surfaces of the notch at incident angles smaller than those of ions that directly bombard the same surfaces. Thus, in spite of the energy loss during the scattering event, scattered $\mathrm{Cl}$ atoms can still contribute to etching of the notch surfaces.

Charging of the exposed $\mathrm{SiO}_{2}$ surface causes forward ion deflection which contributes profoundly to notching. The local electric field distribution in the notch region is determined by solving the Laplace equation subject to the boundary conditions outlined in Sec. IV B. This procedure is repeated for every new cell position reached along the $\mathrm{SiO}_{2}$ surface by the evolving notch apex. Since the bottom surface is discretized by 100 cells, this implies that the Laplace equation, subject to the changing boundary condition described by Eq. (4.4), will be solved 50 times to reach a depth of $50 \%$ of the poly-Si line thickness. The new electrostatic force field obtained at each step is used for ion trajectory calculations to advance the notch boundary to the next cell layer.

In the following subsections, notch profiles are presented as a function of three parameters: $E_{\mathrm{th}}, \mu$, and $\theta_{\mathrm{cr}}$. The scattering parameters refer only to the interaction of $\mathrm{Cl}^{+}$with the $\mathrm{SiO}_{2}$ surface. Comparisons will reveal how these parameters influence the notch shape and depth.

\section{Notch evolution and the importance of forward ion deflection and scattering}

We start by comparing an experimental profile to the simulated notch profile obtained by using the measured $E_{\mathrm{th}}=10 \mathrm{eV},{ }^{12}$ while fixing the scattering parameters at reasonable values $\left(\mu=1.0\right.$ and $\left.\theta_{\mathrm{cr}}=45^{\circ}\right)$. The experimental notch profile published in the work of Nozawa et al. [see their Fig. 10(c)] for an open area width of $2 \mu \mathrm{m}$, is digitized and shown by the open circles in Fig. 13. The result of the simulation with full forward ion deflection and scattering is shown by the superimposed continuous line. $18.7 \times 10^{6}$ ions per $0.5 \mu \mathrm{m}$ along the lower sheath boundary were required for this calculation; this number corresponds to the experi- 


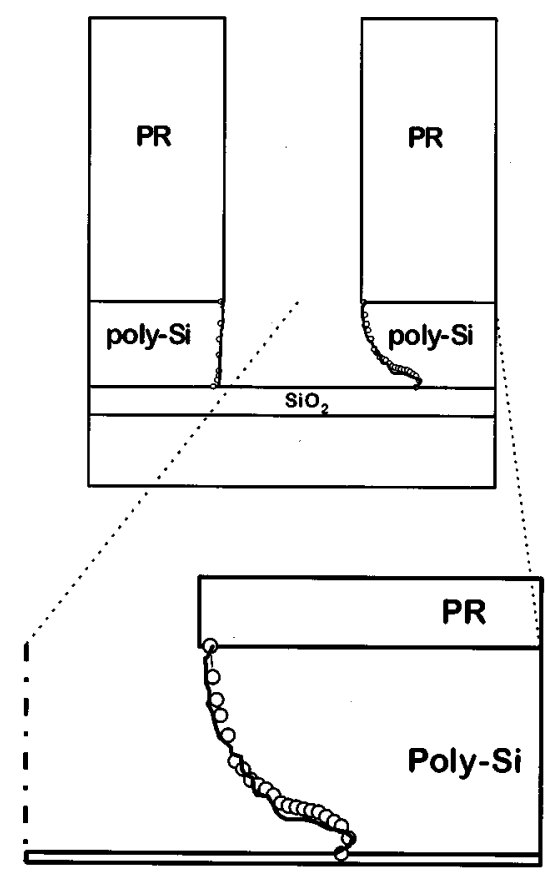

FIG. 13. Comparison between experimental and simulated notch profiles. The circles represent the digitized experimental profile of Nozawa et al. (Ref. 1) for an open area of $2 \mu \mathrm{m}$, etched under the plasma conditions given in Sec. II. The notch region has been expanded to facilitate clearer comparison. The poly-Si dimensions in the expanded region are to scale, unlike the PR dimensions.

mental $200 \%$ overetching time for the purposes of the simulation. All essential features are captured with no parameter adjustment. The contribution of scattering to reactive collisions is only $8.6 \%$. It will be shown later that better matching of the notch apex is possible by reducing the values of $\mu$ and $\theta_{\mathrm{cr}}$. However, lower values of these parameters do not seem to be physical and would detract from the appeal of comparing a fixed model prediction to the complex notch profile. Moreover, it must be noted that spontaneous etching, which has been neglected in this calculation, could easily account for the small mismatch. Deep notching occurs at the inner side of the outermost feature, while only inward tapering is seen for the neighboring poly-Si sidewall. The outer poly-Si sidewall does not develop a notch during overetching; ions get repelled from the sidewall towards the bottom surface because of a repulsive potential condition for ions. the poly-Si line is at a higher potential $(+7.8 \mathrm{~V})$ than that of the neighboring open area (as low as $+3 \mathrm{~V}$ near the center, see Fig. 8). The tapered profile of the outer sidewall, seen in the experimental micrograph [Fig. 10(c) of Ref. 1], is believed to form during the standard etch. When poly-Si is still present (uncleared) in the open area, the sidewalls are at the same potential with the open area; therefore, the potential condition that steers ions away from the sidewall does not yet exist. Ions get bent towards and bombard the outer poly-Si sidewall as they cross the strong negative potential near the upper photoresist sidewall.

To better delineate the contribution of the different mechanisms to notching, we present in Figs. 14(a) and 14(b)

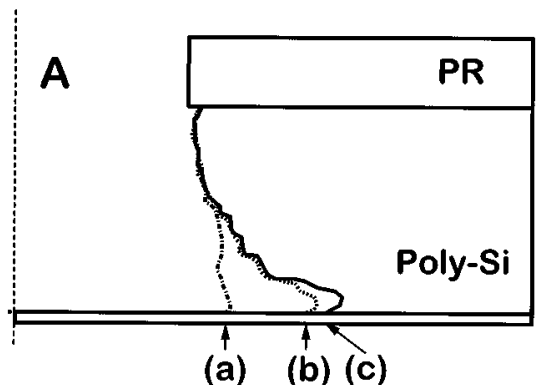

(a) (b) (c)

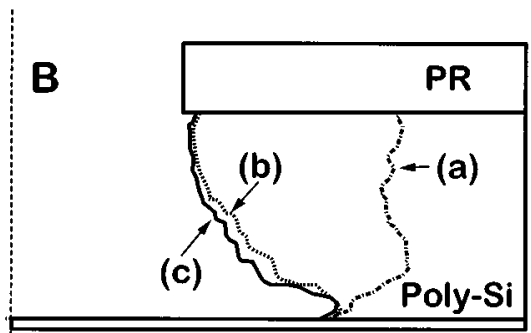

FIG. 14. Comparison of sidewall profiles when various contributing mechanisms to etching are considered. (A) Profiles generated for $200 \%$ overetching time and (B) profiles allowed to reach the same depth as the experimental notch profile, thus requiring different overetching times. (a) Only deflection due to open area charging is allowed, (b) forward ion deflection due to charging of the exposed $\mathrm{SiO}_{2}$ is included, and (c) forward scattering on the exposed $\mathrm{SiO}_{2}$ is also included. The PR is not to scale.

the expanded notch profiles for a fixed overetching time and for a fixed notch depth, respectively. All profiles are generated for $E_{\mathrm{th}}=10 \mathrm{eV}$. When neither forward ion deflection nor forward scattering on the $\mathrm{SiO}_{2}$ surface are considered, only ions that get deflected in the trench area and actually impact the poly-Si sidewall may react with a probability given by Eq. (4.1). These ions cause inward tapering of the sidewall, as illustrated by the profile (a) of Fig. 14(A). If this profile were allowed to evolve to the experimental notch depth [Fig. 14(B), profile (a)] by increasing the overetching time, a shape significantly different from the experimental profile would develop. When forward ion deflection is included (forward scattering is still excluded), the notch shape is more reasonable [see profile (b) in Figs. 14(A) and 14(B)]. Reactive collisions increase significantly $(55 \%)$ from profile (a) to (b) of Fig. 14(A). Clearly, the effect of local charging of the exposed $\mathrm{SiO}_{2}$ surface is dramatic. However, matching of the experimental profile is still not satisfactory. Direct inelastic scattering on the $\mathrm{SiO}_{2}$ surface compensates for the small deviation in depth, as shown by profile (c) in Figs. 14(A) and 14(B) (identical to the profile of Fig. 13). This profile was generated by setting $\mu=1.0$ and $\theta_{\mathrm{cr}}=45^{\circ}$. For the same number of impacting ions, the notch is deeper and, overall, the profile is a better depiction of the observed notch. Scattering increases the number of reactive collisions by an additional $8.6 \%$. Note that the scattering contribution is limited to the notch apex. This result is not surprising in view of the inefficient energy transfer for wide angle scattering. Thus, neargrazing collisions yield the most energetic scattered projectiles, which predominantly bombard the notch apex. But where do the ions that scatter on the $\mathrm{SiO}_{2}$ surface come 
from? Ions that approach the bottom at a small distance from the poly-Si sidewall encounter a strong repulsive potential (see Fig. 9) and will get deflected. There is a variety of bent trajectories from a few degrees all the way to right angles, guaranteeing a supply of ions that will bombard every newly exposed $\mathrm{SiO}_{2}$ surface segment at progressively increasing angles of incidence. The latter condition is further enhanced by the significant positive charging of the $\mathrm{SiO}_{2}$ surface that gets exposed early in the etching process. Notching does not occur at other sidewalls of the microstructure because of the lack of a steep potential gradient that could impart large translational energy to the deflected ions.

Ion trajectory bending due to local electric fields and the resulting direct bombardment of the poly-Si sidewall foot is clearly the dominant mechanism by which notching develops. However, when scattering effects are considered, the significant notch depth and shape are predicted with greater precision, which suggests that direct inelastic scattering is an important mechanism for notching. Direct inelastic scattering was found to be uniquely responsible for microtrenching in hyperthermal neutral beam etching. ${ }^{10}$ A notch is indeed reminiscent of a sharp microtrench (with a $90^{\circ}$ clockwise rotation) and both phenomena have a common origin in the forward scattering of energetic particles, albeit the contribution to notching is smaller. Thus, no new mechanism is required to explain notching. All profiles presented in the following sections will include scattering effects.

\section{E. Parameter sensitivity analysis}

\section{Notching dependence on $E_{t h}$}

The energy threshold for reaction influences profoundly both the shape and extent of the notch. A value of $E_{\text {th }}$ larger than the maximum translational energy that can be gained by ions as they cross the charging potential gradient between the trench bottom and the poly-Si line, will produce minimal or no notching. Some inward tapering of the sidewall foot is, however, still expected to occur as this phenomenon is caused by the more energetic ions that arrive near the poly-Si sidewall and their trajectories get only slightly bent. The threshold dependence is systematically investigated in Fig. 15 , where we plot notch profile sequences as a function of $E_{\text {th }}$ while keeping the scattering parameters constant at $\mu=1.0$ and $\theta_{\mathrm{cr}}=45^{\circ}$. The five profiles of each sequence correspond to specific notch depths in increments of $10 \%$, up to $50 \%$ of the poly-Si line thickness, so that the notch shape evolution can be compared. It is noted that an exponentially increasing overetching time is required to reach the same notch depth as $E_{\text {th }}$ increases. The width of the notch opening decreases with $E_{\mathrm{th}}$, a result of the diminishing ion deflection contributions to etching. Indeed, for reaction to occur at larger $E_{\mathrm{th}}$, the ions must be accelerated to larger final energies which can only be attained closer to the bottom of the trench, where the potential gradient to the sidewall foot is the largest. Notice that for $E_{\mathrm{th}}=20 \mathrm{eV}$, the notch is significantly narrower. At even larger values of $E_{\text {th }}$, very thin notches (not

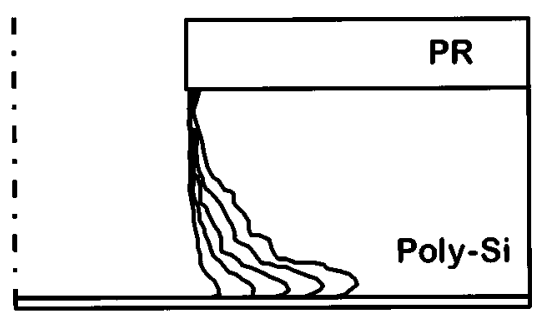

i (a)

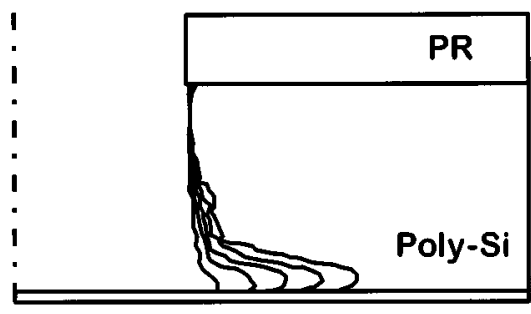

i (b)

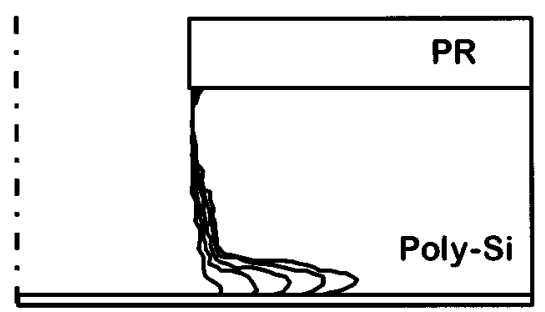

i(c)

FIG. 15. Notch profile dependence on the energy threshold for reaction shown for (a) $E_{\mathrm{th}}=10 \mathrm{eV}$; (b) $E_{\mathrm{th}}=15 \mathrm{eV}$; (c) $E_{\mathrm{th}}=20 \mathrm{eV}$. The profile sequences correspond to increments in depth of $10 \%$ of the poly-Si linewidth. An exponentially increasing overetching time was required to reach the same notch depth for larger $E_{\mathrm{th}}$; that is, in the overetching time required to obtain the deepest notch for $E_{\mathrm{th}}=10 \mathrm{eV}$, only a minute notch (approx. the first profile) would develop for $E_{\mathrm{th}}=20 \mathrm{eV}$. All profiles were generated by including all three contributing mechanisms described in Fig. 1. The scattering parameters were $\mu=1.0, \theta_{\mathrm{cr}}=45^{\circ}$. The PR is not to scale.

shown) can be formed, requiring a significantly increased overetching time. Very few scattering collisions contribute to etching at these large threshold energies.

\section{Notching dependence on $\mu$}

The ratio $\mu$ of the ion mass to the effective mass of the surface $\left(\mathrm{Cl}\right.$-covered $\left.\mathrm{SiO}_{2}\right)$ affects critically the energy transfer during a collision event. Small values of $\mu$ lead to inefficient energy transfer, thereby producing scattered projectiles with large final energy. Thus, decreasing values of $\mu$ make the scattering contributions to notching more significant. However, the relatively small number of ions that penetrate the repulsive electrostatic potential to impinge on the exposed $\mathrm{SiO}_{2}$, where they may undergo scattering, reduces the influence of $\mu$ on the notch profile. This result is illustrated in Fig. 16, where profiles, generated by the same number of ions $\left(18.7 \times 10^{6}\right)$, are compared for three different values of $\mu$. The other parameters were: $E_{\mathrm{th}}=10 \mathrm{eV}$ and $\theta_{\mathrm{cr}}=45^{\circ}$. There are small changes to the notch depth and 


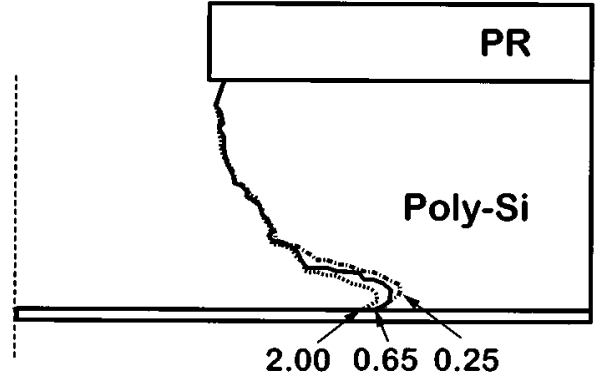

FIG. 16. Notch profile dependence on $\mu$. The profiles shown were generated with the same number of ions $\left(18.7 \times 10^{6}\right), E_{\mathrm{th}}=10 \mathrm{eV}$ and $\theta_{\mathrm{cr}}=45^{\circ}$. The numbers in the figure correspond to the different values of $\mu$. The PR is not to scale.

shape for a decrease in the value of $\mu$ from 2.0 to 0.25 , which corresponds to a dramatic change in the nature of the scattering surface. The changes in the profile are bearly discernible for $\mu$ between 0.5 and 1.0 ; these values are believed to be more realistic for a $\mathrm{Cl}$-covered $\mathrm{SiO}_{2}$ surface. The value of $\mu=1.0$, used in the scattering model, corresponds to binary collisions of $\mathrm{Cl}^{+}$with chemisorbed $\mathrm{Cl}$ atoms. The lack of sensitivity can easily be explained by the fact that mostly grazing collisions are contributing to etching of the notch apex; for such collisions, energy transfer is small and only weakly dependent on $\mu$. Interestingly, improved fitting of the experimental notch apex (see Fig. 13) can be obtained for $\mu=0.25$. However, such a low value of $\mu$ is believed not to be physical for the system under study.

\section{Notching dependence on $\boldsymbol{\theta}_{c r}$}

The critical angle $\theta_{\mathrm{cr}}$ corresponds to the onset of direct inelastic scattering and affects notching by changing the number of projectiles that scatter forward. For example, decreasing the value of $\theta_{\mathrm{cr}}$ from $60^{\circ}$ to $30^{\circ}$ allows more ions (those that impinge at $\theta_{i}$ between $30^{\circ}$ and $60^{\circ}$ ) to undergo direct inelastic scattering. However, these ions result in reflected projectiles with lower energy, a consequence of the smaller incident/reflection angles involved. Thus, the influence of $\theta_{\text {cr }}$ on notching will be negligible for large values of $E_{\text {th }}$ and $\mu$ and more important at smaller values of these parameters. However, even in the latter case, the relatively small number of ions undergoing scattering makes the notching dependence on $\theta_{\mathrm{cr}}$ insignificant. For the case of Fig. 13, where $E_{\mathrm{th}}=10 \mathrm{eV}$ and $\mu=1.0$, the total number of reactive collisions due to scattering increased from $6.3 \%$ to $9.3 \%$ when decreasing $\theta_{\text {cr }}$ from $60^{\circ}$ to $30^{\circ}$, respectively. The differences in notch shape and depth were imperceptible [a minuscule increase in notch depth is expected for small $\theta_{\text {cr }}$ due to the increased scattering probability, see Eq. (4.2)]. The notch profile's lack of sensitivity to $\theta_{\text {cr }}$ combined with the weak dependence on $\mu$ render our simulation virtually parameter independent.

\section{F. Notching dependence on the open area width}

We have seen that the outermost poly-Si line acquires a larger potential than the neighboring open area, thus attract-

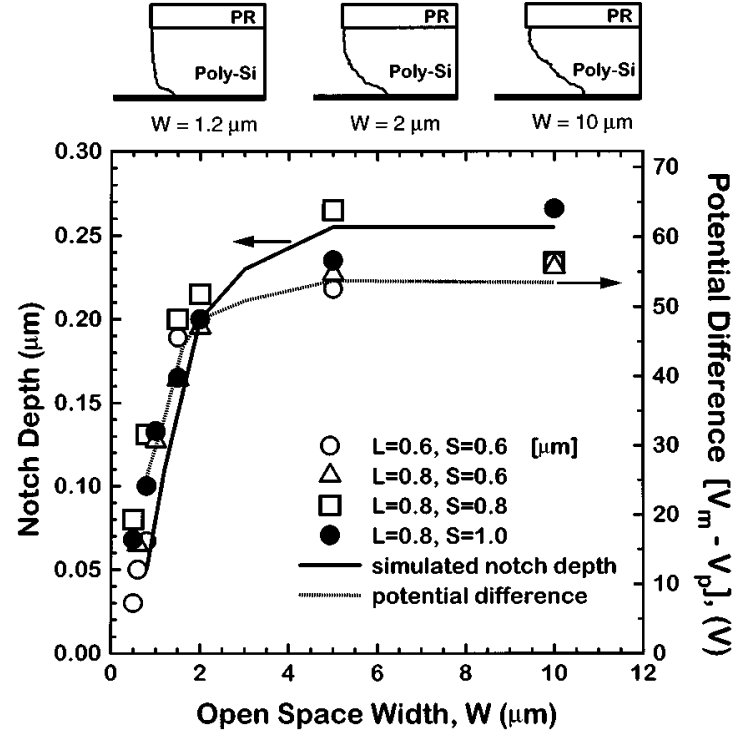

FIG. 17. Dependence of the calculated and experimental notch depth on the open space width (see Fig. 3 for definitions). The points are reproduced from the work of Nozawa et al. (Ref. 1) and describe measurements of notch depth as a function of open space width for experiments with different line-and-space structures ( $L=$ linewidth, $S=$ spacewidth), etched under the plasma conditions described in Sec. II. Also shown is the difference between the calculated maximum of the steady-state potential distribution at the trench bottom surface $\left(V_{m}\right)$ and the equipotential of the outermost polysilicon line $\left(V_{p}\right)$. The inset figures illustrate the notch profile for the indicated open area widths obtained for the same $200 \%$ overetching time.

ing more electrons. It is easily inferred from Fig. 8 that, as the width of the open area decreases, the potential at its entrance becomes slightly more negative. The electron flux towards the bottom decreases as more electrons are repelled back into the plasma. Moreover, electron shadowing increases. Fewer electrons arrive at the poly-Si sidewall, which leads to a larger potential there and less directional bending of the ion trajectories in the trench. Thus, the notching effect should decrease with the width of the open area and become insignificant when the width becomes equal to the space between lines for the given electron temperature. On the other hand, as the open area width increases beyond $2 \mu \mathrm{m}$ (see Fig. 8), more electrons penetrate towards the bottom. These electrons cannot be attracted to the sidewall because the latter is now located further away from the centerline. Most of the electrons go towards the bottom to neutralize the larger flux of ions there, rather than towards the sidewalls. The reduction in the potential of the poly-Si line will cease, thus quenching the rate of notch deepening.

To quantify this effect, we have calculated the potential contour maps and performed full notching simulations for various open area widths $(W)$. The model parameters remained unchanged at $E_{\mathrm{th}}=10 \mathrm{eV}, \mu=1.0$, and $\theta_{\mathrm{cr}}=45^{\circ}$. In all cases, the notch depth was calculated for the same (200\%) overetching time. The results are plotted in Fig. 17 (continuous solid line) as a function of the open area width. Four sets of experimental data obtained by Nozawa et al. ${ }^{1}$ are reproduced in the same figure. Although the points were obtained for various line and space widths, they show the same de- 
pendence on open area width. The simulation captures almost quantitatively the observed behavior, an accomplishment that further supports the soundness of the mechanisms and effects considered. We have also plotted the difference, $\Delta V=V_{m}-V_{p}$, between the maximum value $\left(V_{m}\right)$ of the potential distribution along the trench bottom and the equipotential of the outermost poly-Si line $\left(V_{p}\right)$ for the various open area widths, appropriately scaled on the right axis of Fig. 17. This potential difference tracks the experimental results remarkably well. This observation is perhaps not surprising. Not only is the potential difference largely responsible for ion deflection near the poly-Si sidewall foot but it also determines the energy of the deflected ions. Thus, a correlation between this potential difference and the notch depth is expected to exist. We note that the potential distribution in the trench region remains unchanged for open area widths greater than $5 \mu \mathrm{m}$. Thus, the notch profile for $W=10$ $\mu \mathrm{m}$ is identical to the one obtained for $W=5 \mu \mathrm{m}$. Our findings discredit the conjecture that electron accumulation at the outermost line is determined solely by the solid angle available to electrons to reach the poly-Si sidewall, ${ }^{16}$ which predicts that the notch depth should increase continuously with the open space width.

\section{G. Notching in electrically connected poly-Si lines}

When the poly-Si conductivity is high and the lines are electrically connected, notches form at all lines. ${ }^{1,16}$ The notch depth depends weakly on the total poly-Si perimeter bordering the open area; a three order-of-magnitude change in the perimeter doubles the notch depth under identical etching conditions. ${ }^{1}$ Our $2 \mathrm{D}$ simulation cannot be used to predict quantitatively the effect of the perimeter, unless the lines are connected through the substrate. However, it is instructive to extend the simulation to describe the electron redistribution over connected lines and investigate why the notch forms on both sides of the trench. Assuming that all four lines of the grating to the left of a $10 \mu \mathrm{m}$ open area (see Fig. 3) are connected, the charge redistribution must occur along all poly-Si surfaces so that all lines reach the same potential. This task is easily performed by the technique presented in Sec. III D. In all other respects, the microstructure charging proceeds in identical fashion to the case of nonconnected lines. When steady-state charging is reached, the potential distribution in the trench is found to be bimodal, as shown in Fig. 18. The potential maxima appear near the sidewall foot of both poly-Si lines. A small difference between the left $(55.4 \mathrm{~V})$ and right $(54.3 \mathrm{~V})$ peak potentials is believed to be a result of statistical fluctuations. Since both the left and right poly-Si lines are at equipotential $(9.5 \mathrm{~V})$, the potential gradient towards either of the poly-Si sidewalls is virtually identical.

The symmetric potential distribution predicts symmetric notches at both lines. Since the difference between the $\mathrm{SiO}_{2}$ peak potential and the poly-Si equipotential $(55.4-9.5=45.9$ $\mathrm{V})$ is smaller than in the nonconnected case $(60-6.3=53.7$ V) for equal open area width, the notch should be more shallow than the one observed in Fig. 18 (inset profile for

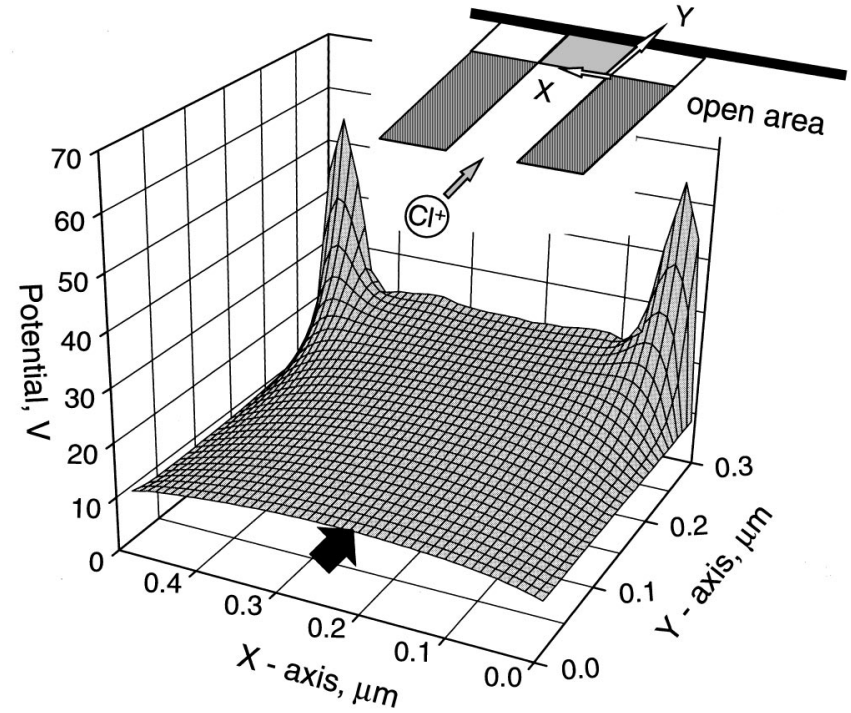

FIG. 18. The 2D potential distribution in the trench area bordered by the poly-Si sidewalls for the case of electrically connected lines next to a $10 \mu \mathrm{m}$ open area. The inset illustrates the area of interest and defines the origin for the potential surface. The arrows show the direction of ions as they approach the potential surface. Note the differences with the potential surface for the case of nonconnected lines (Fig. 9).

$W=10 \mu \mathrm{m})$ for the same overetching time. In addition, the upper poly-Si sidewalls should etch less given the smaller inclination of the potential surface.

The predicted profile, plotted in Fig. 19, has been obtained for $200 \%$ overetching time by following the procedures of Sec. IV C with the fixed parameters $\left(E_{\mathrm{th}}=10 \mathrm{eV}\right.$, $\mu=1.0$, and $\theta_{\mathrm{cr}}=45^{\circ}$ ). The notches are fairly symmetric and the predictions for their depth and shape hold. The digitized experimental profile obtained by Nozawa et al. [Fig. 1(c) of Ref. 1] is also plotted on the same figure. The matching is impressive, considering that the exact experimental overetching time was used for the simulation rather than letting the simulated profile evolve to the experimental notch depth. This observation suggests that the differences in the geometry between the experimental and simulated structures are not very important for notching. Indeed, the notch depth dependence on perimeter is weak (vide supra), and the potential distribution in the trench for a simulated open area width

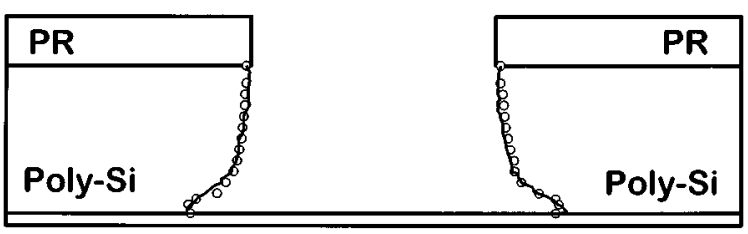

FIG. 19. Comparison between experimental and simulated notch profiles for the case of electrically connected lines. The circles represent the digitized experimental profile of Nozawa et al. (Ref. 1) for the last trench before a large open area, etched under the plasma conditions given in Sec. II. The simulated profile was generated for $200 \%$ overetching time with the potential distribution of Fig. 18. The notch region has been expanded to facilitate clearer comparison. The poly-Si and trench dimensions are to scale; the PR has been truncated. 
of $10 \mu \mathrm{m}$ is not expected to differ significantly from that for a widely open area (see Sec. IV F). The ability of the simulation to capture quantitatively the experimental notch characteristics in the case of connected lines increases our confidence that the dominant notching mechanisms have been considered and that the simple models used to describe them are sound.

\section{IS REDUCTION OF NOTCHING POSSIBLE?}

There are two ways to approach the question of notching reduction in high density plasmas. First, the plasma characteristics ( $\mathrm{rf}$ bias and frequency, electron temperature, plasma density) may be changed so that the ion flux and/or the magnitude of the ion trajectory bending towards the sidewall foot are reduced. For example, Fujiwara et $a .^{2}{ }^{2}$ demonstrated that a reduction in the electron temperature reduces notching, which they attributed to a diminishing supply of electrons to the outer poly-Si sidewall. They have also found that, for a given (and not too large) electron temperature, plasma power and $\mathrm{rf}$ bias, there exists a window of rf frequencies where notching can be eliminated. Increasing the rf frequency (up to a point) shifts the IEDF to a larger average energy; ${ }^{7}$ thus, Fujiwara et al. conjectured that this effect was similar to that achieved by increasing the rf bias, namely, the incident ions become more energetic which enhances their "surface charge neutralization', properties (?). However, such solutions may compromise etch rate (former) or selectivity (latter) and may be undesirable. Moreover, the proposed explanations of the influence of the plasma parameters on notching should be considered with caution; for example, the reduction of the notch depth with increasing rf bias can be simply explained by the increase in the vertical etch rate, which reduces the overetching time.

Second, the chemistry of etching and/or the scattering dynamics may be modified to reduce the reactivity of the ions and atoms impinging on the sidewall foot. Based on the elucidation of the notching mechanisms accomplished in the present study, several variations of this approach can be devised.

The threshold energy dependence suggests at least two ways to minimize notching: (a) by changing the nature of the etched surface, and (b) by decreasing the surface temperature. For example, addition of $\mathrm{O}_{2}$ to the plasma leads to the formation of $\mathrm{SiO}_{x} \mathrm{Cl}_{y}$ species on the surface, which are important in suppressing the etching of $\mathrm{Si}\left(E_{\mathrm{th}} \uparrow\right) .{ }^{17}$ Lowering the surface temperature decreases the volatility of reaction by products and could also change the mechanism by which etching proceeds, again decreasing the etch rate. In spite of the fact that these effects also occur at the uncleared poly-Si at the bottom of narrow trenches, etching of these surfaces can be accomplished by the higher energy ions of the IEDF, whose trajectories are not altered by the local electric fields. An optimum $\mathrm{O}_{2}$ percentage (in the reactor gas feed) and an optimum wafer temperature may exist such that the deflected low energy ions etch the poly-Si sidewall foot at a negligible rate, whereas the undeflected higher energy ions, that di- rectly bombard the uncleared poly-Si at the trench bottom, continue to etch at an acceptable rate.

It is interesting to note that a less dense chlorine plasma should result in more $\mathrm{Cl}_{2}^{+}$. A larger $E_{\text {th }}$ of about $25 \mathrm{eV}$ has been reported for etching and sputtering of a chlorinated $\mathrm{Si}$ surface with a mixed beam of $\mathrm{Cl}_{2}^{+}: \mathrm{Cl}^{+}$of approximately $7: 1 .{ }^{15}$ In addition, $\mathrm{Cl}_{2}^{+}$scattering on the $\mathrm{Cl}$-covered $\mathrm{SiO}_{2}$ should almost double the value of $\mu$. These two observations strongly suggest that a reduction in notching is possible when the molecular ion content of the plasma increases. Decreasing the plasma power should be an easy way to accomplish the latter, although the effect may not be unambiguous given the simultaneous reduction in plasma density. Notice again that clearing of the poly-Si at the bottom of narrow trenches should still be feasible with the higher energy ions of the IEDF, albeit at a lower etch rate. This model prediction could help reduce the notching problem in chlorinebased poly-Si etch processes.

The results on the threshold energy dependence also describe the consequences of using different ions to etch. For example, $\mathrm{Ar}^{+}$has an energy threshold of about $45 \mathrm{eV}^{15}$ and an atomic mass not very different from that of $\mathrm{Cl}^{+}$. If the $\mathrm{SiO}_{2}$ surface continues to be covered by chlorine (separate gas feed?), then the value of $\mu$ will not be significantly different. Thus, our calculations suggest that notching will not evolve beyond the initial inward tapering, if an Ar plasma with similar characteristics (density, bias, rf frequency, $T_{e}$ ) is used and provisions are made to saturate the wafer surface with chlorine. Notice that an IEDF for $\mathrm{Ar}^{+}$, similar to the one in Fig. 4(a), guarantees etching during the overetching step to clear the trench bottom surfaces, since ions with energy larger than threshold are still available.

Finally, the lower reactivity of heavier halogen atoms toward poly-Si suggests that switching, for example, to a $\mathrm{Br}^{+}$-containing plasma during overetching should reduce notching, mainly because of the larger energy threshold, but also because of less energetic scattered atoms in view of their larger atomic mass $(\mu \uparrow)$.

\section{CRITICAL EVALUATION OF KEY ASSUMPTIONS}

Perhaps the most severe assumption in this simulation is the use of the potential boundary condition expressed by Eq. (4.4), which is based only on ion accumulation at the exposed $\mathrm{SiO}_{2}$ surface. Electrons could also be attracted to the same surface, reducing the net charge accumulation there. The deviation is expected to be more significant near the initial position of the sidewall, because it is more difficult for electrons to reach deep into the notch. A reduction in the potential would lead to a narrower notch as it has been found for the extreme case of a constant potential equal to $V_{p}$ (independent of the position along the $\mathrm{SiO}_{2}$ surface). However, the very large positive potential of the trench bottom, with a maximum of $58.7 \mathrm{~V}$ only $40 \mathrm{~nm}$ away from the initial sidewall foot, is expected to significantly hinder the penetration of electrons into the etched area. Therefore, we believe that the exponential decrease in potential with distance into the notch, expressed by the proportionality to $N(i)$, is very rea- 
sonable; it certainly does a good job in predicting the notch depth and shape and the open area dependence.

Charging of the microstructure surfaces is essential to the appearance of the notching effect. Surface and tunneling currents and secondary electron emission can reduce the charge on insulator surfaces, thereby altering the electrostatic force field responsible for trajectory bending. In the absence of any measurements reported in the literature on the mobility of electrons and ions on photoresist and adsorbate-covered $\mathrm{SiO}_{2}$ surfaces, these currents have been neglected. Tunneling through the $\mathrm{SiO}_{2}$ is known to occur for relatively thin oxide layers, ${ }^{18}$ but a thick layer should prevent such a current. Arnold and Sawin ${ }^{4}$ suggested the use of $1000 \mathrm{~V} / \mu \mathrm{m}$ as a reasonable upper bound for the sustainable potential difference along the surface of $\mathrm{SiO}_{2}$. Our maximum steady-state potential gradient was calculated to be slightly larger at 1270 $\mathrm{V} / \mu \mathrm{m}$. However, this value is based on the original position of the sidewall. As the notch begins to evolve, the poly-Si sidewall gets further away from the position of the potential maximum at the $\mathrm{SiO}_{2}$ surface, thus yielding considerably smaller surface potential gradients. Nevertheless, larger charging potentials will be calculated for the surfaces of thick insulator layers by neglecting surface currents and secondary electron emission. A reduced surface charge on the $\mathrm{SiO}_{2}$ will allow lower energy ions to make it further into the trench before their trajectories are bent towards the sidewalls. Because the IEDF is broad with a large number of ions at intermediate energies [between the two peaks, see Fig. 4(a)], there will always be ions whose trajectories will be bent to directly bombard the poly-Si sidewall foot and to impact the $\mathrm{SiO}_{2}$ surface at large angles of incidence. If the resulting translational energies are still above $E_{\mathrm{th}}$, notching will develop, but it may not be as severe as in the full charging case. In this respect, our simulation may overestimate the extent of notching.

While ions enter the sheath during all phases of the rf cycle, electrons penetrate in bursts and only during the sheath potential minimum. This results in surface potentials that vary periodically with the rf frequency. Our assumption that ions and electrons impinge on the microstructure sequentially yields the average surface charging potentials. While this procedure does not reflect the realistic process, the time-varying component is small and does not significantly change the steady-state average potentials which required $3500 \mathrm{rf}$ cycles to develop. The notch evolution calculation is only slightly sensitive to this small potential variation.

We also have omitted the possible deposition of etch inhibitors that may protect the sidewalls. From the point of view of our simulation, inhibitor deposition corresponds to an increase in the effective energy threshold for reaction. We have found that the upper portion of the poly-Si sidewalls is bombarded by ions which have been decelerated significantly by the positive potential of the bottom surface. Thus, any inhibitor deposition in the poly-Si sidewalls should reduce sidewall etching. However, if there is an angular dependence to the inhibitor deposition, then the sidewall foot may have a thinner inhibitor layer that can easily be broken through by the more energetic ions that impinge at that location. This situation could lead to narrow thin notches, which evolve parallel to the $\mathrm{SiO}_{2}$ surface mainly due to forward inelastic scattering.

Considering only specular direct inelastic scattering (reflection) at the $\mathrm{SiO}_{2}$ surface may lead to underestimating the notch depth and width. In modeling microtrenching, we have used a Gaussian function to describe forward scattering with an incident-angle-dependent variance. ${ }^{10}$ Such broad forward scattering would result in more energetic atoms ejected at super-specular angles, thus enhancing notching very close to the poly-Si/ $\mathrm{SiO}_{2}$ interface. In the absence of angularly resolved $\mathrm{Cl}$-atom flux distributions for the inelastic scattering of $\mathrm{Cl}^{+}$on $\mathrm{Cl}$-covered $\mathrm{SiO}_{2}$, consideration of the broad scattering requires a new adjustable parameter, limiting the scope of our simple model.

As it has been presented, our simulation captures several observations relating to the notching effect while keeping the number of parameters to a minimum. In fact, we have examined the effects of plasma characteristics ( $\mathrm{rf}$ bias, $\mathrm{rf}$ frequency, electron temperature, and plasma density) on notching and predicted the experimental trends without changing the model parameters. These results will be published elsewhere.

\section{SUMMARY AND CONCLUSIONS}

We have presented a 2D numerical simulation of microstructure charging, ion trajectory bending, scattering, and reactions occurring during the overetching step of silicon-oninsulator structures. The simulation offers insight into the plausible mechanisms responsible for the "notching" effect. Notching was found to have its origin in local electric-fieldinduced trajectory bending of energetic ions that (a) bombard directly and etch the poly-Si sidewall foot, and (b) first undergo forward scattering or electrostatic deflection at the newly exposed $\mathrm{SiO}_{2}$ surface under the notch, resulting in energetic projectiles that subsequently impinge onto and etch the notch surfaces. Nonuniform local electric fields capable of deflecting ions form as a result of differential charging in high aspect ratio structures, which is induced by the directionality difference between ions and electrons. Notching depends strongly on the magnitude of the potential gradient that develops in the vicinity of the inner side of the outermost poly-Si line. When the poly-Si lines are electrically connected, similar potential gradients develop by the sidewall foot of all grating lines, leading to notching everywhere. The elucidation of the mechanisms responsible for notching allowed prediction of new ways to reduce or eliminate the effect, based on a modification of the chemistry of etching and/or the scattering dynamics.

As these results were based on simple electrostatics and scattering dynamics, one could argue that the notching effect could have been predicted before it was ever seen in experiments. This realization bears promise for the potential impact of feature-scale modeling on alleviating the problems of future generations of devices. 


\section{ACKNOWLEDGMENTS}

The authors are grateful to J. W. Coburn and V. M. Donnelly for insightful suggestions and a critical review of the manuscript. They would also like to thank V. Vahedi for sharing his knowledge of plasma sheaths and proposing the decaying dipole field explanation. This work was partially supported by Caltech start-up funds and by a NSF Career Award to K. P. G. (CTS-9623450).

${ }^{1}$ T. Nozawa, T. Kinoshita, T. Nishizuka, A. Narai, T. Inoue, and A. Nakaue, Jpn. J. Appl. Phys. 34, 2107 (1995).

${ }^{2}$ N. Fujiwara, T. Maruyama, and M. Yoneda, Jpn. J. Appl. Phys. 34, 2095 (1995); 35, 2450 (1996).

${ }^{3}$ K. K. Chi, H. S. Shin, W. J. Yoo, C. O. Jung, Y. B. Koh, and M. Y. Lee, Jpn. J. Appl. Phys. 35, 2440 (1996).

${ }^{4}$ J. C. Arnold and H. H. Sawin, J. Appl. Phys. 70, 5314 (1991).

${ }^{5}$ H. Ootera, T. Oomori, M. Tuda, and K. Namba, Jpn. J. Appl. Phys. 33, 4276 (1994)

${ }^{6}$ T. Kinoshita, M. Hane, and J. P. McVittie, J. Vac. Sci. Technol. B 14, 560 (1996).
${ }^{7}$ M. S. Barnes, J. C. Foster, and J. H. Keller, IEEE Trans. Plasma Sci. 19, 240 (1991).

${ }^{8}$ M. A. Lieberman, IEEE Trans. Plasma Sci. 16, 638 (1988).

${ }^{9}$ J. R. Woodworth, M. E. Riley, D. C. Meister, B. P. Aragon, M. S. Le, and H. H. Sawin, J. Appl. Phys. 80, 1304 (1996).

${ }^{10}$ G. S. Hwang, C. M. Anderson, M. J. Gordon, T. A. Moore, T. K. Minton, and K. P. Giapis, Phys. Rev. Lett. 77, 3049 (1996).

${ }^{11}$ C. Steinbrüchel, Appl. Phys. Lett. 55, 1960 (1989).

${ }^{12}$ J. P. Chang and H. H. Sawin, J. Vac. Sci. Technol. A (submitted).

${ }^{13}$ E. A. Ogryzlo, D. E. Ibbotson, D. L. Flamm, and J. A. Mucha, J. Appl. Phys. 67, 3115 (1990).

${ }^{14} \mathrm{~J}$. Harris, in Dynamics of Gas-Surface Interactions, edited by C. T. Rettner and M. N. Ashfold (Royal Society of Chemistry, Cambridge, 1991), Chap. 1.

${ }^{15}$ M. Balooch, M. Moalem, W. E. Wang, and A. V. Hamza, J. Vac. Sci. Technol. A 14, 229 (1996).

${ }^{16}$ S. Ogino, N. Fujiwara, H. Miyatake, and M. Yoneda, Jpn. J. Appl. Phys. 35, 2445 (1996).

${ }^{17}$ C. C. Cheng, K. V. Guinn, V. M. Donnelly, and P. I. Herman, J. Vac. Sci. Technol. A 12, 2630 (1994).

${ }^{18}$ K. Hashimoto, Jpn. J. Appl. Phys. 33, 6013 (1994). 